\title{
Self-assembly of surfactants in a supercritical solvent from lattice Monte Carlo simulations
}

\author{
Martin Lísal, ${ }^{\text {a) }}$ Carol K. Hall, and Keith E. Gubbins \\ Department of Chemical Engineering, North Carolina State University, Raleigh, \\ North Carolina 27695-7905 \\ Athanassios Z. Panagiotopoulos \\ Department of Chemical Engineering, Princeton University, Princeton, New Jersey 08544-5263
}

(Received 31 August 2001; accepted 24 October 2001)

\begin{abstract}
We modify Larson's lattice model [J. Chem. Phys. 83, 2411 (1985)] and use it to study self-assembly of surfactants in a supercritical solvent by large-scale Monte Carlo simulations. Carbon dioxide and perfluoroalkylpoly(ethylene oxide) serve as prototypes for the solvent and surfactant, respectively. Larson-model type parameters for carbon dioxide and perfluoroalkylpoly(ethylene oxide) are obtained using experimental values of critical parameters and solubility along with a modified Berthelot combining rule. We perform canonical Monte Carlo simulations at a supercritical temperature, varying the number of surfactant head and tail segments, the solvent density and the surfactant concentration. Various properties such as the critical micelle concentration, the aggregate size distribution, and the size and shape of the micelles are evaluated and pseudophase diagrams are constructed. We further investigate the ability of the surfactant solutions to dissolve more solute than solutions without surfactants by calculating the partition coefficient. Water serves as a prototype for the solute and Larson-model type parameters for water are obtained in the same way as for carbon dioxide and perfluoroalkylpoly(ethylene oxide). (C) 2002 American Institute of Physics. [DOI: 10.1063/1.1428347]
\end{abstract}

\section{INTRODUCTION}

Supercritical fluids are becoming increasingly important solvents in polymer science and engineering. ${ }^{1,2}$ Supercritical carbon dioxide $\left(\mathrm{scCO}_{2}\right)$, in particular, is a widely used solvent due to its low cost, moderate critical conditions $\left(T_{C}\right.$ $=304.13 \mathrm{~K}, P_{C}=73.773 \mathrm{bar}$ ), and environmentally benign nature. However, several important classes of substances such as water, hydrophilic substances, and most polymers exhibit very low solubility in $\mathrm{scCO}_{2}$. This has led to an extensive search for surfactants with the ability to solubilize such substances in $\mathrm{scCO}_{2} \cdot{ }^{3}$ Surfactant molecules used in $\mathrm{scCO}_{2}$ have two mutually incompatible components: a $\mathrm{CO}_{2}$-philic tail with an affinity for $\mathrm{CO}_{2}$ molecules and a $\mathrm{CO}_{2}$-phobic head with a repulsion to $\mathrm{CO}_{2}$ molecules. When immersed in $\mathrm{scCO}_{2}$ these surfactants self-assemble and form micelles with the $\mathrm{CO}_{2}$-phobic head in the core. These micelles can be employed to enhance the solubility of normally insoluble compounds by incorporating them into their core, a process known as micellar solubilization.

A common feature of the surfactants used in $\mathrm{scCO}_{2}$ is that they contain tails with fluorinated $\left(\mathrm{CF}_{3}, \mathrm{CF}_{2}\right)$ segments. The properties of fluorinated compounds are distinctly different from those of their hydrocarbon analog due to their relatively large size and the highly electronegative nature of the fluorine atom. ${ }^{4}$ This is manifested, for example, by the high

${ }^{a)}$ On leave of absence from E. Hála Laboratory of Thermodynamics, Institute of Chemical Process Fundamentals, Academy of Sciences of the Czech Republic, 16502 Prague 6, Czech Republic. solubility of fluorocarbons in $\mathrm{scCO}_{2}$, in contrast to their hydrocarbon analog. 5

During the last decade, there has been a huge experimental effort devoted to the study of the formation of micelles in $\mathrm{scCO}_{2} \cdot{ }^{3}$ In comparison there have been only a few attempts to use molecular-level approaches to understand the process of micellization in $\mathrm{scCO}_{2}$. Salaniwal et al. ${ }^{6-8}$ studied selfassembly of micelles in $\mathrm{scCO}_{2}$-dichain surfactant-water systems by molecular dynamics simulations with an atomistic model. Baysal et al. ${ }^{9}$ performed molecular dynamics calculations with an atomistic model to study the conformational properties of single chain diblocks of poly(1,1dihydroperfluorooctyl acrylate) and poly(vinyl acetate) in $\mathrm{scCO}_{2}$. Luna-Bárcenas et al. ${ }^{10}$ used the statistical associating fluid theory ${ }^{11}$ to model phase behavior in $\mathrm{scCO}_{2}$-poly(FOA) systems and Colina et al. ${ }^{12}$ combined the osmotic pressure approach with the statistical associating fluid theory to study phase diagrams of copolymers in $\mathrm{scCO}_{2}$.

The time scale for the assembly of equilibrium surfactant structures is currently beyond the reach of atomistic simulations for many surfactant solutions of interest. This has led to the introduction of coarse-grained models in which a number of atoms are grouped together and are represented in a simplified manner. The two main families of coarse-grained models employed are off-lattice models and lattice models. The former are typically more versatile and realistic while the latter are usually more computationally efficient. ${ }^{13}$ Larson's model of water-amphiphile-oil systems ${ }^{14}$ is typical of the many lattice models available. It uses a fully occupied cubic lattice with lattice points representing either a solvent 
molecule, a solute molecule or a surfactant segment. An entity on a given lattice point interacts with nearest neighbor and diagonal nearest neighbor points, resulting in a coordination number of 26. No interactions at greater distance are present. Larson's model simplifies molecular interactions in water-amphiphile-oil systems by assuming that the contact energies of lyophilic and hydrophilic units are the same regardless of which of the lyophilic units (tail or oil), or which of the hydrophilic units (head or water) are interacting. Monte Carlo simulations conducted on Larson's model have been able to qualitatively describe different phases in wateramphiphile-oil systems. ${ }^{15}$

The objective of this work is to study self-assembly of surfactants in supercritical solvent and micellar solubilization in supercritical solvent-surfactant systems by performing Monte Carlo simulations on a modified version of Larson's lattice model. We use carbon dioxide, perfluoroalkylpoly(ethylene oxide) and water as prototypes for the solvent, surfactant and solute, respectively. We chose perfluoroalkylpoly(ethylene oxide): $\mathrm{F}\left(\mathrm{CF}_{2}\right)_{n}\left(\mathrm{CH}_{2} \mathrm{CH}_{2} \mathrm{O}\right)_{m} \mathrm{H}(m$ $=4-9, n=7-11 ; m<n)$ as the prototypical nonionic surfactant because: (i) it is linear and easy to model, (ii) it has a $\mathrm{CO}_{2}$-philic tail: $\mathrm{F}\left(\mathrm{CF}_{2}\right)_{n}$, and (iii) its head, $\left(\mathrm{CH}_{2} \mathrm{CH}_{2} \mathrm{O}\right)_{m} \mathrm{H}$, interacts favorably with water molecules. Moreover, Fulton et al. ${ }^{16}$ have already shown that this surfactant forms micelles in $\mathrm{scCO}_{2}$.

In the remainder of this paper, we first introduce a modified version of Larson's model (Sec. II) and show how to estimate Larson-model type parameters for carbon dioxide, perfluoroalkylpoly(ethylene oxide) and water (Sec. III). This is followed by a description of simulation methodology and by a definition of quantities that characterize the micellization process (Sec. IV). Section V presents and discusses the results for the critical micelle concentration $(\mathrm{cmc})$, the aggregate size distribution, the size and shape of the micelles and the partition coefficient as well as resulting pseudophase diagrams. We conclude with a summary in Sec. VI.

\section{MODEL}

Our lattice model is a modification of Larson's model ${ }^{14}$ which treats a supercritical solvent-surfactant-solute system as a ternary mixture of $N_{s}$ monomer solvent $\left(\mathrm{CO}_{2}\right)$ molecules $(s), N_{l}$ monomer solute $\left(\mathrm{H}_{2} \mathrm{O}\right)$ molecules $(l)$ occupying one lattice point each, and $N_{A}$ surfactant chain molecules occupying $r$ lattice points each on a lattice with coordination number $z=26$. We denote the surfactant as $H_{m} T_{n}$, where $H$ stands for a head segment (either a $\mathrm{CH}_{2} \mathrm{CH}_{2} \mathrm{O}$ or a $\mathrm{CH}_{2} \mathrm{CH}_{2} \mathrm{OH}$ group) and $T$ for a tail segment (either a $\mathrm{CF}_{2}$ or a $\mathrm{CF}_{3}$ group); $r=m+n$.

In contrast to Larson's model, our lattice is not fully occupied but instead contains $N_{v}$ vacancies $(v)$. This allows us to study micellar behavior as a function of the solvent density. Each molecular unit $(s, l, T$, and $H)$ interacts with the other molecular units with a contact energy $\varepsilon_{a b}(a, b$ $=s, l, T, H)$. Negative values of $\varepsilon_{a b}$ correspond to attractive interactions while nonnegative values of $\varepsilon_{a b}$ represent repulsive interactions. It should also be mentioned that the vacancies are treated as molecular units in our model with $\varepsilon_{v b}$ ( $b=s, l, T, H, v)$ equal to zero by definition. ${ }^{17}$
When dealing with mixtures and their properties, the real interest is in the thermodynamic functions of mixing, i.e., in the values of functions relative to their values for the pure fluids. The thermodynamic mixing functions such as the free energy of mixing depend on the interchange energies, $w_{a b}$, which are linear combinations of contact energies ${ }^{18}$ as

$$
w_{a b}=\varepsilon_{a b}-\frac{\varepsilon_{a a}+\varepsilon_{b b}}{2} \quad(a, b=s, l, T, H, v \text { with } a \neq b) .
$$

Instead of $w_{a b}$ lattice fluid theories usually employ the Flory-Huggins interaction parameters, $\chi_{a b},{ }^{19}$ defined to be

$$
\chi_{a b}=\frac{z}{k_{B} T} w_{a b} \quad(a, b=s, l, T, H, v \text { with } a \neq b),
$$

where $T$ is the temperature and $k_{B}$ is Boltzmann's constant.

\section{PARAMETER ESTIMATION}

\section{A. Like contact energies}

The contact energies between like molecular units are obtained by matching the critical temperatures of the model fluids with the critical temperatures of the real fluids. A monomer on a lattice with coordination number $z=26$ is the model fluid for carbon dioxide and water. For the nonionic surfactant $\mathrm{F}\left(\mathrm{CF}_{2}\right)_{n}\left(\mathrm{CH}_{2} \mathrm{CH}_{2} \mathrm{O}\right)_{m} \mathrm{H}(m=4-9, n=7-11 ; m$ $<n$ ), we consider the tail and head interactions separately and make the usual assumption that the interaction parameters of a particular molecular group can be transferred to different molecules. ${ }^{20}$ The contact energies between tail-tail and head-head segments are found by matching the critical temperatures of chains of lengths $n$ and $m$ (on the lattice with $z=26$ ) with the critical temperatures of $\mathrm{C}_{n} \mathrm{~F}_{2 n+2}$ and $\mathrm{H}\left(\mathrm{CH}_{2} \mathrm{CH}_{2} \mathrm{O}\right)_{m} \mathrm{H}$, respectively.

We utilize the following experimental values for the critical temperatures: $T_{C}=304.13 \mathrm{~K}$ for carbon dioxide, $T_{C}=647.10 \mathrm{~K}$ for water and $\left\{\left(n, T_{C}\right)\right\}=\{(7,475.3 \mathrm{~K})$, $(8,498.2 \mathrm{~K}), \quad(9,521.2 \mathrm{~K}),(10,542.35 \mathrm{~K}),(11,559.2 \mathrm{~K})\}$ for $\mathrm{C}_{n} \mathrm{~F}_{2 n+2} \cdot{ }^{21}$ Since the experimental critical temperatures for $\mathrm{H}\left(\mathrm{CH}_{2} \mathrm{CH}_{2} \mathrm{O}\right)_{m} \mathrm{H}$ are not known, we estimate them by the Fedors group-contribution method. ${ }^{21}$ The estimation gives: $\left\{\left(m, T_{C}\right)\right\}=\{(4,713 \mathrm{~K}), \quad(5,754 \mathrm{~K}), \quad(6,790 \mathrm{~K}), \quad(7,821 \mathrm{~K})$, $(8,848 \mathrm{~K}),(9,872 \mathrm{~K})\}$. The critical temperatures of the model fluids

$$
T_{C}^{*} \equiv \frac{k_{B} T_{C}}{w_{v a}}=-\frac{2 k_{B} T_{C}}{\varepsilon_{a a}}
$$

are available from lattice Gibbs ensemble Monte Carlo simulations: ${ }^{22} \quad T_{C}^{*}=11.7$ for monomers and $\left\{\left(r, T_{C}^{*}\right)\right\}$ $=\{(4,19.9), \quad(5,21.3), \quad(6,22.3), \quad(7,23.2), \quad(8,23.9)$, $(9,24.6),(10,25.2),(11,25.7)\}$ for chains of length $r$.

Comparing the $T_{C}$ values for monomers with those for pure $\mathrm{CO}_{2}$ and $\mathrm{H}_{2} \mathrm{O}$ we find that the solvent-solvent $\left(\mathrm{CO}_{2}-\mathrm{CO}_{2}\right)$ and solute-solute $\left(\mathrm{H}_{2} \mathrm{O}-\mathrm{H}_{2} \mathrm{O}\right)$ contact energies are $\varepsilon_{s s} / k_{B}=-52 \mathrm{~K}$ and $\varepsilon_{l l} / k_{B}=-111 \mathrm{~K}$, respectively. The tail-tail and head-head segment contact energies obtained for different chain lengths are plotted in Fig. 1. Since they 


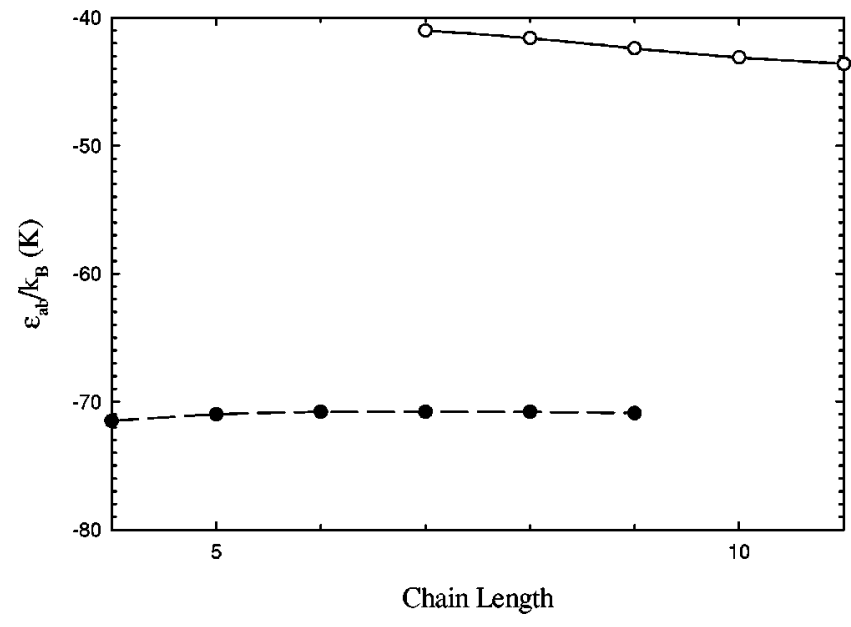

FIG. 1. The surfactant tail-tail $(\bigcirc,-)$ and head-head $(\bullet,---)$ segment contact energies $\varepsilon_{a b} / k_{B}$ for different chain lengths; $k_{B}$ is Boltzmann's constant.

depend only slightly on the chain length we average them over the chain length to obtain final values for the tail-tail and head-head segment contact energies of $\varepsilon_{T T} / k_{B}$ $=-42 \mathrm{~K}$ and $\varepsilon_{H H} / k_{B}=-71 \mathrm{~K}$, respectively. All like contact energies are summarized in Table I.

\section{B. Unlike contact energies}

The contact energies between the unlike molecular units cannot be obtained simply from the standard Berthelot combining rule, ${ }^{23}$ since this is for repulsion and dispersion interactions, whereas our contact energies include contributions from electrostatic and other forces. Its use would result in having large solubilities for water in $\mathrm{scCO}_{2}$ or in having a weak attractive water-surfactant head interaction, in contrast to what is known empirically. Generally-speaking, the character and strength of the interactions between two components are established by the sign and magnitude of the Flory-Huggins interaction parameters $\chi_{a b} \cdot{ }^{24}$ Negative values of $\chi_{a b}$ correspond to electrostatic or hydrogen bonding interactions between unlike species while small and positive values of $\chi_{a b}$ characterize dispersion interactions. For two mutually incompatible (insoluble) components $\chi_{a b}$ is generally large and positive (e.g., $\chi \approx 5$ for water-oil systems at room temperature). ${ }^{24,25}$

TABLE I. The contact energies $\varepsilon_{a b} / k_{B}$ and associated values of the FloryHuggins interaction parameters $\chi_{a b}$ at a temperature of $350 \mathrm{~K}$ for the lattice model of supercritical solvent-surfactant-solute systems studied; $k_{B}$ is Boltzmann's constant.

\begin{tabular}{lcllcc}
\hline \hline \multicolumn{2}{c}{ Like interactions } & & \multicolumn{3}{c}{ Unlike interactions } \\
\cline { 1 - 2 } \cline { 1 - 1 } Type of interaction & $\varepsilon_{a b} / k_{B}(\mathrm{~K})$ & & Type of interaction & $\varepsilon_{a b} / k_{B}(\mathrm{~K})$ & $\chi_{a b}$ \\
\hline solvent-solvent & -52 & & solvent-solute & -20 & 4.6 \\
solute-solute & -111 & & solvent-tail & -44 & 0.2 \\
tail-tail & -42 & & solvent-head & 0 & 4.6 \\
head-head & -71 & & solute-tail & -10 & 4.9 \\
& & & solute-head & -101 & -0.7 \\
& & head-tail & -49 & 0.6 \\
\hline \hline
\end{tabular}

We use the following strategy for determining the unlike contact energies: (i) we adjust the contact energies $\varepsilon_{a b}$ between mutually incompatible molecular units [solvent-solute $\left(\mathrm{CO}_{2}-\mathrm{H}_{2} \mathrm{O}\right)$, solvent-head segment $\left(\mathrm{CO}_{2}-\mathrm{CH}_{2} \mathrm{CH}_{2} \mathrm{O}\right.$ or $\left.\mathrm{CO}_{2}-\mathrm{CH}_{2} \mathrm{CH}_{2} \mathrm{OH}\right)$, and solute-tail segment $\left(\mathrm{H}_{2} \mathrm{O}-\mathrm{CF}_{2}\right.$ or $\left.\mathrm{H}_{2} \mathrm{O}-\mathrm{CF}_{3}\right)$ ] to roughly represent experimental solubility data, and (ii) we modify the standard Berthelot combining rule to reflect the character and strength of the interactions between the mutually compatible molecular units [solventtail segment $\left(\mathrm{CO}_{2}-\mathrm{CF}_{2}\right.$ or $\left.\mathrm{CO}_{2}-\mathrm{CF}_{3}\right)$, solute-head segment $\left(\mathrm{H}_{2} \mathrm{O}-\mathrm{CH}_{2} \mathrm{CH}_{2} \mathrm{O}\right.$ or $\left.\mathrm{H}_{2} \mathrm{O}-\mathrm{CH}_{2} \mathrm{CH}_{2} \mathrm{OH}\right)$, and head segmenttail segment $\left(\mathrm{CH}_{2} \mathrm{CH}_{2} \mathrm{O}-\mathrm{CF}_{2}\right.$ or $\left.\left.\mathrm{CH}_{2} \mathrm{CH}_{2} \mathrm{OH}-\mathrm{CF}_{3}\right)\right]$.

\section{Contact energies between mutually incompatible molecular units}

We utilize the expression for the Gibbs energy of mixing, $\Delta G_{\text {mix }}$, given by the lattice fluid theory ${ }^{17}$ and the double tangent construction ${ }^{26}$ to calculate the solubility of the solute $\left(\mathrm{H}_{2} \mathrm{O}\right)$ in the solvent $\left(\mathrm{CO}_{2}\right)$, of the head segment $\left[\left(\mathrm{CH}_{2} \mathrm{CH}_{2} \mathrm{O}\right) \mathrm{H}\right]$ in the solvent $\left(\mathrm{CO}_{2}\right)$ and of the tail chains $\left[\mathrm{F}\left(\mathrm{CF}_{2}\right)_{n}\right]$ in the solute $\left(\mathrm{H}_{2} \mathrm{O}\right)$. We vary $\chi_{a b}$ in $\Delta G_{\text {mix }}$ to approximately match these to the experimentally measured solubilities for $\mathrm{H}_{2} \mathrm{O}$ in $\mathrm{scCO}_{2}$, for ethanol in $\mathrm{scCO}_{2}$ and for fluorocarbons in $\mathrm{H}_{2} \mathrm{O}$, respectively, at $T=350 \mathrm{~K}$ and supercritical pressures, resulting in $\chi_{s l}=4.6, \chi_{s H}=4.6$ and $\chi_{l T}$ $=4.9$. (We use the following values of solubilities: ${ }^{27} \sim 2 \%$ for $\mathrm{H}_{2} \mathrm{O}$ in $\mathrm{scCO}_{2}, \sim 1.5 \%$ for ethanol in $\mathrm{scCO}_{2}$, and $\sim 1 \%$ for fluorocarbons in $\mathrm{H}_{2} \mathrm{O}$.) Values of the solvent-solute, solvent-head segment, and solute-tail segment contact energies corresponding to these $\chi_{s l}, \chi_{s H}$, and $\chi_{l T}$ [see Eqs. (2.1) and (2.2)] are $\varepsilon_{s l} / k_{B} \equiv \chi_{s l}(T / z)+\left(\varepsilon_{s s}+\varepsilon_{l l}\right) /\left(2 k_{B}\right)$ $=-20 \mathrm{~K}, \quad \varepsilon_{s H} / k_{B} \equiv \chi_{s H}(T / z)+\left(\varepsilon_{s s}+\varepsilon_{H H}\right) /\left(2 k_{B}\right)=0 \mathrm{~K}$, and $\varepsilon_{l T} / k_{B} \equiv \chi_{l T}(T / z)+\left(\varepsilon_{l l}+\varepsilon_{T T}\right) /\left(2 k_{B}\right)=-10 \mathrm{~K}$, respectively. It is interesting to note that the resulting solventsolute and solute-tail interactions are attractive while the solvent-head interaction is repulsive. However, these attractive interactions are much weaker than the solvent-solvent, solute-solute, and tail-tail attractive interactions.

\section{Contact energies between mutually compatible molecular units}

Applying the standard Berthelot combining rule for calculation of unlike contact energies to our system does not automatically yield the correct sign for the Flory-Huggins interaction parameter $\chi_{a b}$ for mutually compatible molecular units. Therefore, we suggest the following modification of the standard Berthelot combining rule which takes into account the sign of $\chi_{a b}$ :

$$
\varepsilon_{a b}=-\sqrt{\varepsilon^{*} \cdot \varepsilon^{\chi}},
$$

where

$$
\varepsilon^{*}=\frac{\varepsilon_{a a}+\varepsilon_{b b}}{2}
$$

corresponds to the athermal solution $\left(\chi_{a b}=0\right)$ (Ref. 18) and

$$
\begin{aligned}
\varepsilon^{\chi} & =\max \left(\varepsilon_{a a}, \varepsilon_{b b}\right) \quad \text { if } \quad \chi_{a b}>0 \\
& =\min \left(\varepsilon_{a a}, \varepsilon_{b b}\right) \quad \text { if } \quad \chi_{a b}<0 .
\end{aligned}
$$


Use of this modified Berthelot combining rule therefore requires a priori knowledge of the sign of the Flory-Huggins interaction parameter and knowledge of like contact energies.

We assume only dispersion interactions between carbon dioxide and $\mathrm{CF}_{2}$ or $\mathrm{CF}_{3}$ groups as is suggested by the similar solubility parameters for $\mathrm{scCO}_{2}$ and $\mathrm{C}_{6} \mathrm{~F}_{14},{ }^{27}$ and thus $\chi_{s T}$ must be small and positive. On the other hand, we expect hydrogen bonding interactions between water and $\mathrm{CH}_{2} \mathrm{CH}_{2} \mathrm{O}$ or $\mathrm{CH}_{2} \mathrm{CH}_{2} \mathrm{OH}$ groups ${ }^{28}$ and therefore $\chi_{l H}$ must be negative. For interactions between the surfactant tail and head segments, we do not consider electrostatic or hydrogen bonding interactions and so assume that $\chi_{T H}$ is positive.

All unlike contact energies and associated values for the Flory-Huggins interaction parameters at $T=350 \mathrm{~K}$ are summarized in Table I.

\section{Volume of lattice cell}

To complete the description of the lattice model for the supercritical solvent-surfactant-solute systems we need to specify the volume of a lattice cell, $v^{*} \cdot{ }^{17}$ The lattice model assumes that the solvent molecules $\left(\mathrm{CO}_{2}\right)$, surfactant tail segments (either $\mathrm{CF}_{2}$ or $\mathrm{CF}_{3}$ group), and surfactant head segments (either $\mathrm{CH}_{2} \mathrm{CH}_{2} \mathrm{O}$ or $\mathrm{CH}_{2} \mathrm{CH}_{2} \mathrm{OH}$ group), and solute molecules $\left(\mathrm{H}_{2} \mathrm{O}\right)$ are all of the same size. This assumption is reasonable for the solvent molecules, surfactant tail and head segments due to their similar size but is questionable for the solute molecules. However, this drawback cannot be easily overcome in the lattice model used here. Hence, we estimate a value for the volume of the lattice cell by matching the critical volume of the monomer lattice model fluid:22 $\widetilde{v}_{C}$ $\equiv v_{C} /\left(\bar{N}_{\mathrm{A}} v^{*}\right)=2\left(\bar{N}_{\mathrm{A}}\right.$ is Avogadro's constant $)$ with the experimental critical volume of carbon dioxide, ${ }^{21} v_{C}^{\mathrm{CO}_{2}}$ $=94.12 \mathrm{~cm}^{3} / \mathrm{mol}$, resulting in $v^{*}=78.145 \AA^{3}$.

\section{SIMULATION DETAILS}

\section{A. Methodology}

Micellization is a single-phase phenomenon observed at low concentrations of surfactants but above the critical micelle concentration $(\mathrm{cmc})$. This necessitates the use of largescale simulations; our lattices range in size from $50^{3}$ (for higher surfactant concentrations) up to $220^{3}$ (for lower surfactant concentrations) with periodic boundary conditions. These large lattices allow us to have at least five to ten micelles present in the system at any one time, enough to form aggregates typical for the solution in the thermodynamic limit of infinite system size. ${ }^{29}$

We carry out simulations at low surfactant and solute concentrations for supercritical solvent conditions, and therefore the solvent molecules are the abundant species in the simulation box. To reduce CPU time we avoid explicit simulation of the solvent molecules by replacing them with "holes." The contact energies with the solvent molecules $\varepsilon_{a s}$ are set to zero and the rest of the contact energies are rescaled according to the values of the interchange energies $w_{a b}$ defined by Eq. (2.1). Note that the contact energies with the vacancies become nonzero. Thus, only moves for the surfactants, solutes and vacancies need to be performed in the simulations.

The canonical (NVT) Monte Carlo (MC) simulations utilize translation moves and particle interchanges for the monomeric species, and reptation moves and configurational-bias chain regrowth for the surfactants. ${ }^{30}$ The simulations are organized in cycles. Each cycle consists of $N_{v}+r N_{A}$ attempts for the supercritical solvent-surfactant systems or $N_{l}+N_{v}+r N_{A}$ attempts for the supercritical solvent-surfactant-solute systems to move a randomly chosen particle; the types of moves are selected randomly. Note that surfactant moves are attempted with higher frequency than monomeric moves. Configurational-bias and reptation moves are carried out with the same frequency. These result in a very efficient sampling of configurational space. A typical MC run consists of 40000 to 80000 cycles for equilibration (starting from a random configuration) and 40000 cycles for evaluation of quantities of interest. CPU time requirements on a DEC Alpha 533 processor for a typical run are about $4 \mathrm{~h} / 1000$ cycles.

\section{B. Characteristics of the micellization process}

Several properties are calculated during the simulations in order to characterize the micellization process. These properties give information about the $\mathrm{cmc}$, the size and shape of the micelles, and the extent of solubilization. The computational definition of these quantities and the simulation criteria for existence of a micelle are described below.

Any surfactant that contains at least one head segment with a nearest or diagonal nearest neighbor contact to the head segment of another surfactant is considered to be part of a micelle. The aggregation number $M$ is defined to be the number of surfactants contained in the micelle. Calculation of the aggregation number of the micelles is performed by the "cluster multiple labeling technique" of Hoshen and Kopelman. ${ }^{31}$ Surfactants that are not part of micelles are referred to as free unimers.

\section{Critical micelle concentration}

Since there is no true phase transition between the nonaggregated and aggregated (micellar) states, the location of the $\mathrm{cmc}$ depends on the definition used. ${ }^{29}$ We use Israelachvili et al.'s definition of the $\mathrm{cmc}$ as the concentration at which the number of surfactants aggregated in micelles is equal to the number of free unimers. ${ }^{32}$ Other definitions of the $\mathrm{cmc}$ are related to a sharp change either in the solution osmotic pressure ${ }^{33}$ or the polydispersity index, and/or to the first appearance of a peak in the aggregate size distribution as the surfactant concentration increases. ${ }^{34,35}$

\section{Micellar size and shape}

Since micelles are dynamic entities, the aggregation number $M$ is a fluctuating quantity. In general, the micelles are polydisperse and have a distribution of aggregation numbers. Hence, it is convenient to represent the size of a micelle by the number-average aggregation number, 


$$
M_{n}=\frac{\Sigma_{M=2} M x_{M}}{\sum_{M=2} x_{M}}=\frac{\Sigma_{M=2} \Phi_{M}}{\Sigma_{M=2}\left(\Phi_{M} / M\right)},
$$

and the weight-average aggregation number,

$$
M_{w}=\frac{\sum_{M=2} M^{2} x_{M}}{\sum_{M=2} M x_{M}}=\frac{\Sigma_{M=2} M \Phi_{M}}{\sum_{M=2} \Phi_{M}} .
$$

Note that free unimers are not used in calculation of $M_{n}$ and $M_{w}$. In Eqs. (4.1) and (4.2), $x_{M}$ and $\Phi_{M}$ are, respectively, the mole and volume fractions of micelles with aggregation number $M$ and are defined to be

$$
\begin{aligned}
& x_{M}=\frac{N_{M}}{N_{s}+N_{A}}, \\
& \Phi_{M}=\frac{r M N_{M}}{N_{s}+r N_{A}},
\end{aligned}
$$

where $N_{s}$ is equal to the number of solvent molecules, $N_{A}$ is equal to the number of surfactant molecules, and $N_{M}$ is equal to the number of micelles with aggregation number $M$. These fractions are related through

$$
\Phi_{M}=\frac{r M x_{M}}{1+(r-1) x_{A}},
$$

where $x_{A}$ is the surfactant mole fraction. Quantities $x_{M}$ and $\Phi_{M}$ also satisfy a mass balance constraint,

$$
\begin{aligned}
& x_{A}=\sum_{M=1} M x_{M}, \\
& \Phi_{A}=\sum_{M=1} \Phi_{M},
\end{aligned}
$$

where $\Phi_{A}$ is the surfactant volume fraction.

The ratio of $M_{w}$ to $M_{n}$ defines the polydispersity index $I_{p}, 34,35$ which characterizes the width of the aggregate size distribution. If the aggregate size distribution has a micellar peak and the aggregate size distribution is narrow around this peak, $I_{p}$ is close to unity. Aggregate size distributions with a broad distribution around the micellar peak are characterized by large values of $I_{p}$.

To characterize the shape of the micelles we calculate the three principal radii of gyration and asphericity parameter. ${ }^{29,34}$ The three principal radii of gyration, $R_{1}, R_{2}$, and $R_{3}\left(R_{1}\right.$ is the largest radius, followed by $R_{2}$ and then $\left.R_{3}\right)$ are the eigenvalues of the matrix of the radii of gyration. Elements of this matrix are defined to be

$$
R_{\gamma \delta}=\left[\frac{1}{N_{\text {site }}} \sum_{i=1}^{N_{\text {site }}}\left(\gamma_{i}-\gamma_{\text {com }}\right)\left(\delta_{i}-\delta_{\text {com }}\right)\right]^{1 / 2},
$$

where $\gamma_{i}, \delta_{i} \equiv x_{i}, y_{i}, z_{i}$ are the coordinates of surfactant segments in the micelle, $\gamma_{\text {com }}, \delta_{\text {com }} \equiv x_{\text {com }}, y_{\text {com }}, z_{\text {com }}$ are the coordinates of the center-of-mass of the micelle and $N_{\text {site }}$ is the total number of surfactant segments in the micelle. For spherical micelles $R_{1} \approx R_{2} \approx R_{3}$ while for elongated micelles $R_{1}>R_{2} \approx R_{3}$.

The asphericity parameter is defined to be

$$
A_{s}=\frac{\left(R_{1}-R_{2}\right)^{2}+\left(R_{1}-R_{3}\right)^{2}+\left(R_{2}-R_{3}\right)^{2}}{2\left(R_{1}+R_{2}+R_{3}\right)^{2}} .
$$

The asphericity parameter has a value of 0 for spherical micelles and 1 for infinite cylindrical micelles, with intermediate values representing elongated micelles.

\section{Partition coefficient}

The ability of solutions with surfactant to dissolve more solute than solutions without surfactant can be quantified by the partition coefficient,

$$
K=\frac{\Phi_{l}^{A}}{\Phi_{l}^{s}}
$$

where $\Phi_{l}^{A}$ is the solute volume fraction in the solution with surfactant and $\Phi_{l}^{s}$ is the solute volume fraction in the solution without surfactant. The definition of $K$ requires that $\Phi_{l}^{A}$ and $\Phi_{l}^{s}$ be measured under identical conditions, i.e., the volume, temperature, and chemical potentials of solvent and solute must be the same in the solution with and without the surfactant. ${ }^{26}$

The partition coefficient can be measured directly using the two-box simulation method ${ }^{36}$ which is the computational analog of a real osmosis experiment. ${ }^{37}$ The method sets up two simulation boxes: a simulation box with solvent, surfactant, and solute (hereafter called the micelle box) and a simulation box containing only solvent and solute (hereafter called the solvent box). Both boxes are of the same size. Chemical equilibrium for solvent and solute is established by transferring solvent and solute particles between the two boxes. The criteria for the particle exchange (i.e., insertion or particle interchange) are identical to those used in the Gibbs ensemble Monte Carlo simulations. ${ }^{38,39}$ After achieving chemical equilibrium, ensemble averages of the solute volume fractions in the micelle and solvent boxes, $\left\langle\Phi_{l}^{A}\right\rangle$ and $\left\langle\Phi_{l}^{S}\right\rangle$, are used to calculate the partition coefficient according to Eq. (4.8).

It should also be mentioned that the two-box simulation method enables us to avoid simulations in an unstable region. If values of $\left\langle\Phi_{l}^{s}\right\rangle$ are below the solubility limit of the solute in the solvent box, then, due to the established chemical equilibrium for the solvent and solute, it is also assured that the micelle box contains a stable phase. ${ }^{36}$

The solvent box contains a binary mixture of lattice monomeric solvent and solute. As has been shown by Nelson et al. ${ }^{36}$ lattice fluid theory ${ }^{17}$ predicts thermodynamic properties and phase equilibrium of such a mixture within simulation uncertainties. It enables us to replace the solvent box by a "virtual" solvent box where all changes due to the particle exchange are calculated analytically from the lattice fluid theory. The replacement of the solvent box by the virtual solvent box results in significant reduction of computer time. This idea has already been used by Tsangaris and McMahon, ${ }^{40}$ and Mehta and Kofke ${ }^{41}$ in connection with the Gibbs ensemble Monte Carlo simulations. These authors have shown, when formulating the criteria for the particle exchange, that the residual Helmholtz free energy change in the virtual box,

$$
\Delta F_{\text {res }}^{s}=V^{s}\left(f_{\text {res }}^{s, \text { new }}-f_{\text {res }}^{s, \text { old }}\right),
$$


where $V^{s}$ is the volume of the virtual box, $f_{\text {res }}^{s, \text { new }}$ and $f_{\text {res }}^{s, \text { old }}$ are the residual Helmholtz free energy densities of the virtual box in new and old configurations, respectively, plays the role of the intermolecular potential energy change normally used during the particle transfers. We refer readers to the original papers ${ }^{40,41}$ for additional details.

For the case of the binary mixture of lattice monomeric solvent and solute, $f_{\text {res }}^{s}$ needed in Eq. (4.9) is given by the lattice fluid theory ${ }^{17}$ as

$$
\frac{v^{*} f_{\mathrm{res}}^{s}}{k_{B} T}=-\frac{\widetilde{\rho}^{s 2}}{\widetilde{T}}+\left(1-\widetilde{\rho}^{s}\right) \ln \left(1-\widetilde{\rho}^{s}\right),
$$

where

$$
\begin{gathered}
\widetilde{\rho}^{s}=\frac{N^{s} v^{*}}{V^{s}}, \\
\widetilde{T}=\frac{k_{B} T}{\varepsilon^{*}},
\end{gathered}
$$

with

$$
\varepsilon^{*}=-\frac{z}{2}\left(x_{s}^{s 2} \varepsilon_{s s}+2 x_{s}^{s} x_{l}^{s} \varepsilon_{s l}+x_{l}^{s 2} \varepsilon_{l l}\right),
$$

and $N^{s}$ is equal to the number of molecules in the virtual solvent box, and $x_{s}^{s}$ and $x_{l}^{s}$ is equal to, respectively, the solvent and solute mole fractions in the virtual solvent box.

\section{RESULTS AND DISCUSSION}

\section{A. Surfactants self-assembly in the supercritical solvent}

We use three types of surfactant, $\mathrm{H}_{4} \mathrm{~T}_{7}, \mathrm{H}_{4} \mathrm{~T}_{11}$, and $\mathrm{H}_{6} \mathrm{~T}_{11}$, to study the effects of tail- and head-length variation on the micellar behavior in the lattice model systems of $\mathrm{scCO}_{2}-\mathrm{F}\left(\mathrm{CF}_{2}\right)_{n}\left(\mathrm{CH}_{2} \mathrm{CH}_{2} \mathrm{O}\right)_{m} \mathrm{H}$. We carry out NVT MC simulations of the supercritical solvent-surfactant systems at a reduced temperature $T_{r} \equiv T / T_{C}^{\mathrm{CO}_{2}}=1.15$, and reduced solvent densities, $\rho_{r} \equiv v_{C}^{\mathrm{CO}_{2}} / v \quad(v$ is the molar volume of the supercritical solvent-surfactant system), ranging from 1 to 1.82. At each simulated solvent density, the surfactant concentration is varied from $\Phi_{A} \approx 0.1 \%$ to $\Phi_{A} \approx 15 \%$. Typically, we perform two simulations below the cmc and five simulations above the $\mathrm{cmc}$. At surfactant concentrations below the $\mathrm{cmc}$, the aggregate size distribution exhibits only one peak which represents unimers. At surfactant concentrations above the $\mathrm{cmc}$, peaks in the aggregate size distribution form. These peaks and the principal radii of gyration are taken as criteria for the identification of micellar-type aggregates. ${ }^{29}$

Since the simulations are carried out in the canonical ensemble, care must be taken to avoid simulating at state points that fall within an unstable region. If a state point lies within the unstable region, a peak in the aggregate size distribution may form. However such a peak indicates the onset of the formation of an additional phase rather than the formation of a micellar aggregate. As the simulation box size increases, this peak will move toward higher aggregation numbers and become infinite in the thermodynamic limit. In contrast, micellar peaks should stay at approximately the

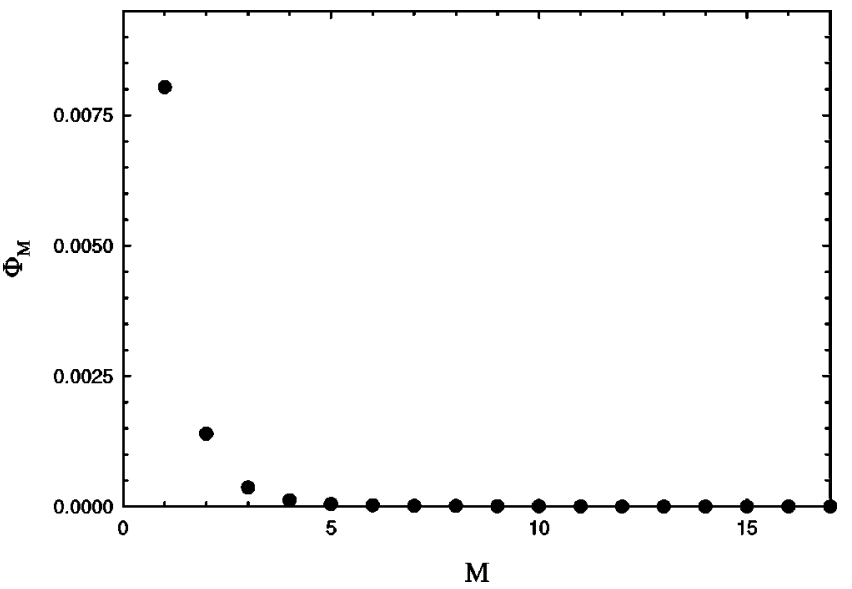

FIG. 2. Aggregate size distribution plotted as volume fraction $\Phi_{M}$ of micelles with the aggregation number $M$ vs $M$ below the critical micelle concentration for $1 \mathrm{vol} \%$ of $H_{4} T_{11}$ in a supercritical solvent at a reduced temperature of 1.15 and a reduced solvent density of 1.400 .

same aggregation number as the simulation box size increases. Similarly, values of the principal radii of gyration characterizing the size and shape of micellar aggregates should be constant as the simulation box size increases since micelles of the same size and shape must form regardless of the size of the simulation box. ${ }^{29}$ Hence, we check to ensure that we are outside an unstable region by repeating some simulations in a larger simulation box to determine if the aggregate size distribution and the principal radii of gyration are fixed. This check is typically performed for each simulated solvent density at a higher surfactant concentration. We usually increase the simulation box by doubling the number of surfactants.

\section{Size and shape of micelles}

Figures 2-6 show typical examples of micelle properties. Figure 2 displays the aggregate size distribution $\Phi_{M}$ vs aggregation number $M$ for $H_{4} T_{11}$ at a state point below the cmc: $\Phi_{A}=1 \%, \rho_{r}=1.400$. The aggregate size distribution exhibits only a unimeric peak and decays sharply to zero for $M>1$. In Fig. 2, we also see that small fractions of dimers and trimers are formed.

Figure 3 presents the aggregate size distribution, and the three principal radii of gyration $\left\{R_{1}, R_{2}, R_{3}\right\}$ and the asphericity parameter $A_{s}$ vs $M$ for $H_{4} T_{11}$ at a state point above the cmc: $\Phi_{A}=2.5 \%, \rho_{r}=1.718$. The aggregate size distribution [Fig. 3(a)] displays a unimeric peak and a micellar peak at $M \approx 55$ and the aggregate size distribution is symmetrical around the micellar peak. The three principal radii of gyration and the asphericity parameter [Fig. 3(b)] suggest that the system contains roughly spherical micelles since $R_{1} \approx R_{2}$ $\approx R_{3}$ and $A_{s} \approx 0$. A peak in $R_{1}$ at $M \approx 85$ has no physical significance (an artifact of simulation) since the aggregate size distribution is virtually zero here.

Figure 4 shows the aggregate size distribution, and $\left\{R_{1}, R_{2}, R_{3}\right\}$ and $A_{s}$ vs $M$ for $H_{4} T_{7}$ at a state point above the cmc: $\Phi_{A}=3.5 \%, \rho_{r}=1.718$. The aggregate size distribution [Fig. 4(a)] exhibits, as in the previous case, a unimeric peak and a micellar peak, now located at $M \approx 90$, but, in contrast 


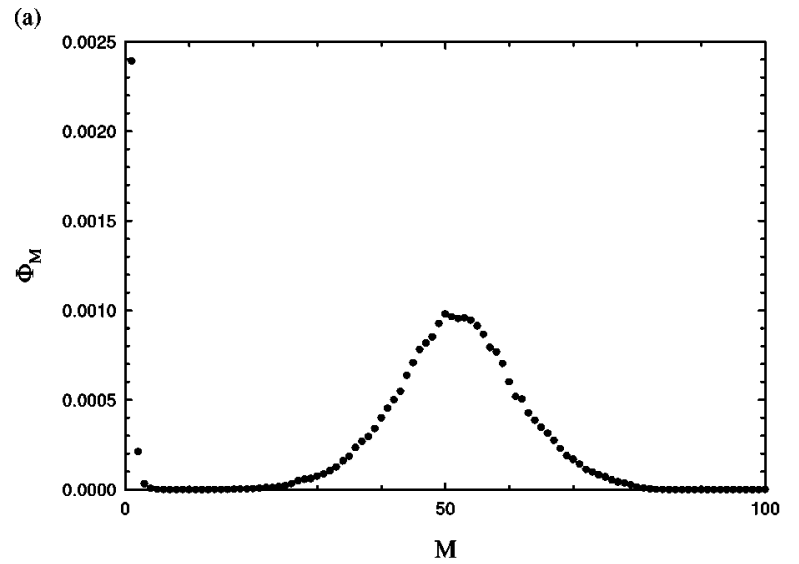

(b)

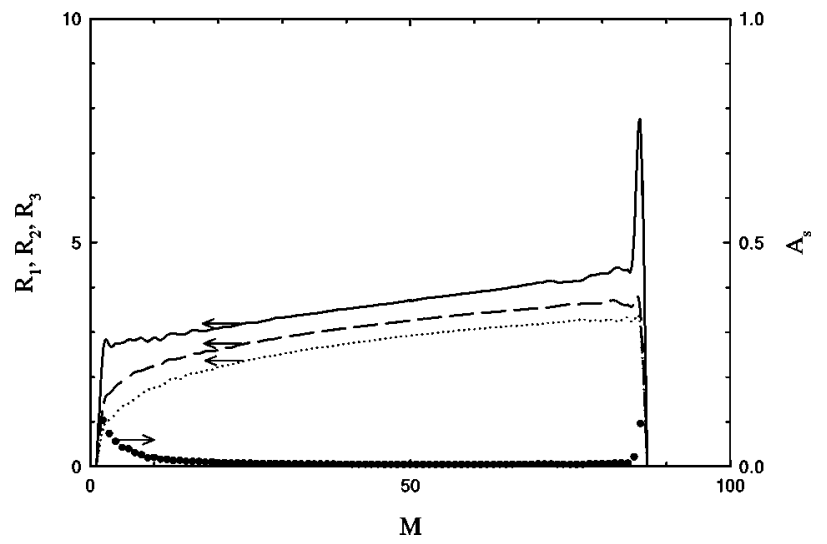

FIG. 3. (a) Aggregate size distribution plotted as volume fraction $\Phi_{M}$ of micelles with the aggregation number $M$ vs $M$, and (b) three principal radii of gyration $R_{1}(-), R_{2}(---), R_{3}(\cdots)$ and asphericity parameter $A_{s}(\bullet)$ vs $M$ above the critical micelle concentration for $2.5 \mathrm{vol} \%$ of $H_{4} T_{11}$ in a supercritical solvent at a reduced temperature of 1.15 and a reduced solvent density of 1.718 .

to the previous case, the aggregate size distribution is asymmetrical around the micellar peak. The asymmetrical behavior is caused by the growth of the micelles at higher $M$ as seen in Fig. 4(b). Figure 4(b) shows that the system contains roughly spherical micelles $\left(R_{1} \approx R_{2} \approx R_{3}\right)$ for $M<110$ and growth of the micelles $\left(R_{1}\right.$ increases in contrast to $R_{2}$ and $R_{3}$ with increasing $M$ ) at higher $M$.

In Fig. 5, we present the aggregate size distribution, and $\left\{R_{1}, R_{2}, R_{3}\right\}$ and $A_{s}$ vs $M$ for $H_{6} T_{11}$ at a high surfactant concentration $\Phi_{A}=15 \%$ and a low solvent density $\rho_{r}$ $=1.000$. The aggregate size distribution [Fig. 5(a)] exhibits additional peaks at high $M$, and $\left\{R_{1}, R_{2}, R_{3}\right\}$ and $A_{s}$ vs $M$ [Fig. 5(b)] show that the system contains both spherical $\left(R_{1} \approx R_{2} \approx R_{3}, A_{s} \approx 0\right)$ and elongated $\left(R_{1}>R_{2} \approx R_{3}, A_{s}>0\right)$ micelles. The first peak, which represents spherical micelles, is located at $M \approx 110$. Other peaks corresponding to elongated micelles are at $M \approx 220,310$, and 390 . These peaks are thought to result from the merging of two or more micelles.

Figure 6 presents the aggregate size distributions for $H_{4} T_{7}$ at high surfactant concentrations $\Phi_{A}=10 \%$ and $\Phi_{A}$ $=15 \%$ and a low solvent density $\rho_{r}=1.000$. The aggregate size distributions display some pronounced peaks but are rather scattered. Moreover, when we repeat the simulations in a larger simulation box the aggregate size distributions
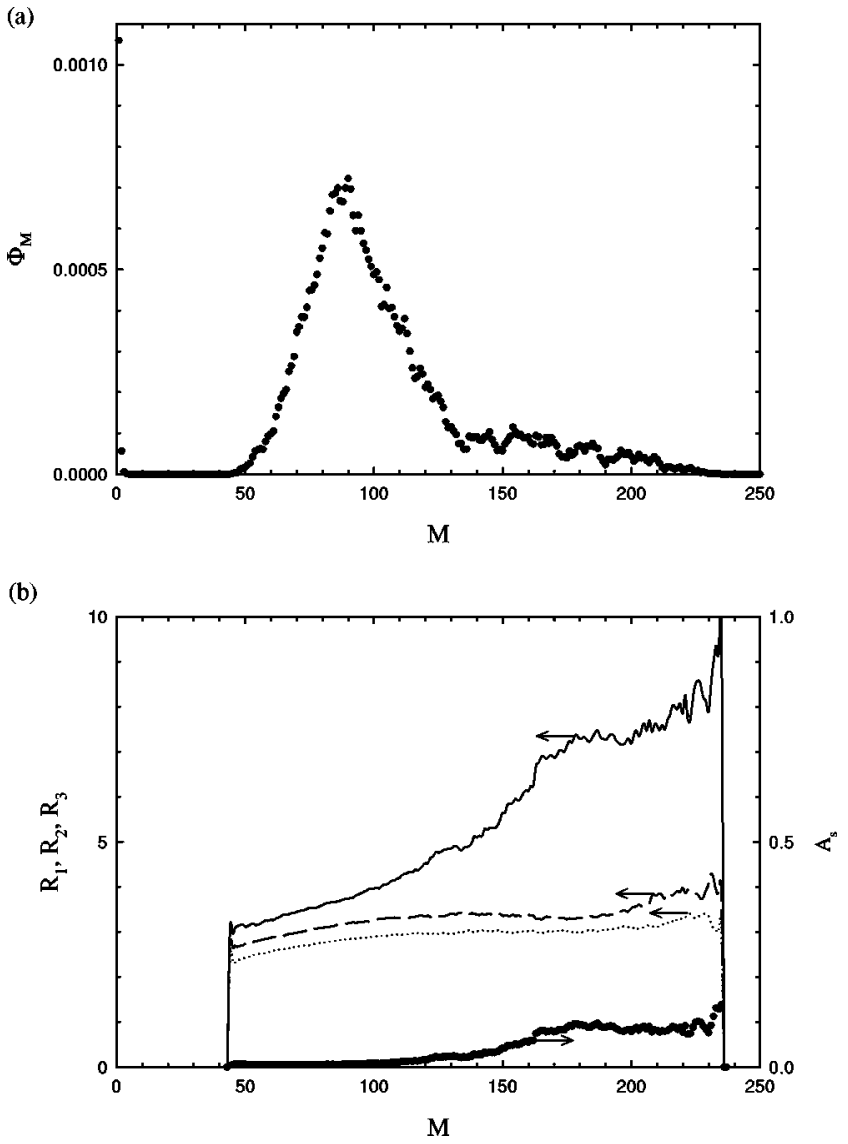

FIG. 4. (a) Aggregate size distribution plotted as volume fraction $\Phi_{M}$ of micelles with the aggregation number $M$ vs $M$, and (b) three principal radii of gyration $R_{1}(-), R_{2}(---), R_{3}(\cdots)$ and asphericity parameter $A_{s}(\bigcirc)$ vs $M$ above the critical micelle concentration for $3.5 \mathrm{vol} \%$ of $H_{4} T_{7}$ in a supercritical solvent at a reduced temperature of 1.15 and a reduced solvent density of 1.718 .

differ from those obtained in a smaller simulation box (not shown here). Finally, visual inspection of simulation snapshots reveals that the simulation boxes contain clusters of random shapes rather than micelles. These characteristics indicate that these simulation state points fall within an unstable region.

Figure 7 shows the effects of variation in the solvent density $\rho_{r}$ on the aggregate size distributions. Figure 7(a) displays the weight-average aggregation number $M_{w}$ vs $\rho_{r}$ and Fig. 7(b) shows the polydispersity index $I_{p}$ vs $\rho_{r}$ for $H_{4} T_{7}$ at $\Phi_{A}=3.5 \%, H_{4} T_{11}$ at $\Phi_{A}=5 \%$, and $H_{6} T_{11}$ at $\Phi_{A}$ $=2.5 \%$. We see from Fig. 7 (a) that $M_{w}$ slightly decreases with decreasing $\rho_{r}$ for $H_{4} T_{7}$ and $H_{4} T_{11}$, and is unaffected by $\rho_{r}$ for $H_{6} T_{11}$. Values of $I_{p}$ [Fig. 7(b)] are close to unity at high $\rho_{r}$ for the three surfactants studied and they increase with decreasing $\rho_{r}$ for $H_{4} T_{7}$ and $H_{4} T_{11}$. For $H_{6} T_{11}$, the values of $I_{p}$ change only marginally with $\rho_{r}$. Recall that values of $I_{p}$ close to unity indicate narrow aggregate size distributions while larger values correspond to broader aggregate size distributions. These trends are a direct consequence of the number of $H$ and $T$ segments in the three surfactants studied. Generally speaking, decreasing $\rho_{r}$ promotes exchange of unimers between the micelles of different $M$ allowing the aggregate size distributions to become 


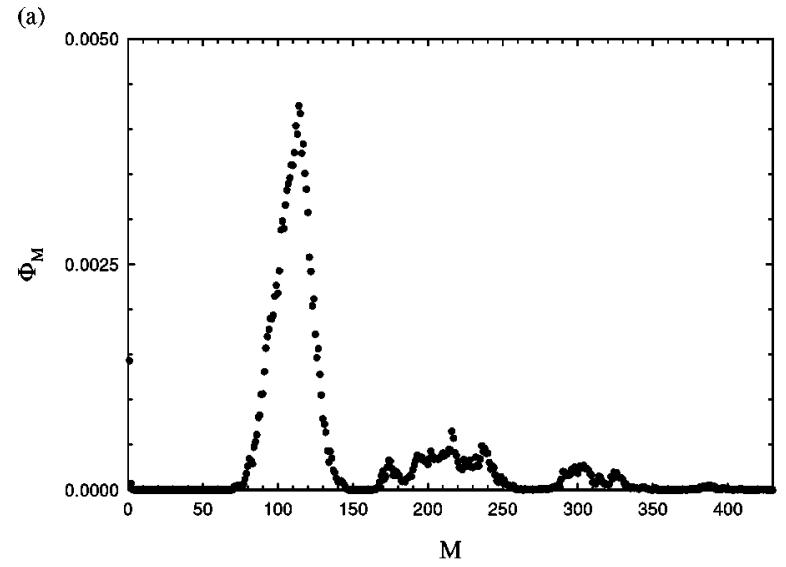

(b)

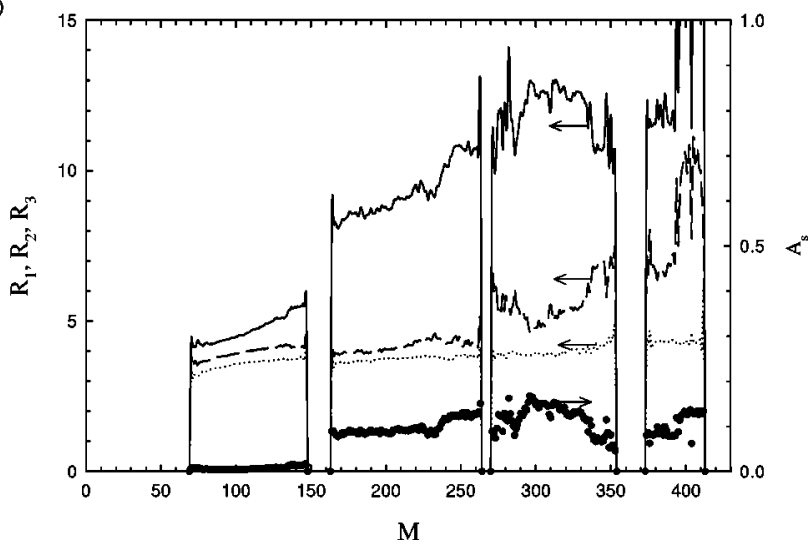

FIG. 5. (a) Aggregate size distribution plotted as volume fraction $\Phi_{M}$ of micelles with the aggregation number $M$ vs $M$, and (b) three principal radii of gyration $R_{1}(-), R_{2}(---), R_{3}(\cdots)$ and asphericity parameter $A_{s}(\bullet)$ vs $M$ above the overlap concentration for 15 vol \% of $H_{6} T_{11}$ in a supercritical solvent at a reduced temperature of 1.15 and a reduced solvent density of 1.000 .

broader. This fact is caused by increasing number of vacancies with decreasing $\rho_{r}$. (Interaction of $H$ segments with vacancies is more favorable than that with solvent molecules since $\chi_{v H}<\chi_{s H}$.) The surfactants with less $H$ segments, $H_{4} T_{7}$ and $H_{4} T_{11}$, enter/leave micelles more easily than the

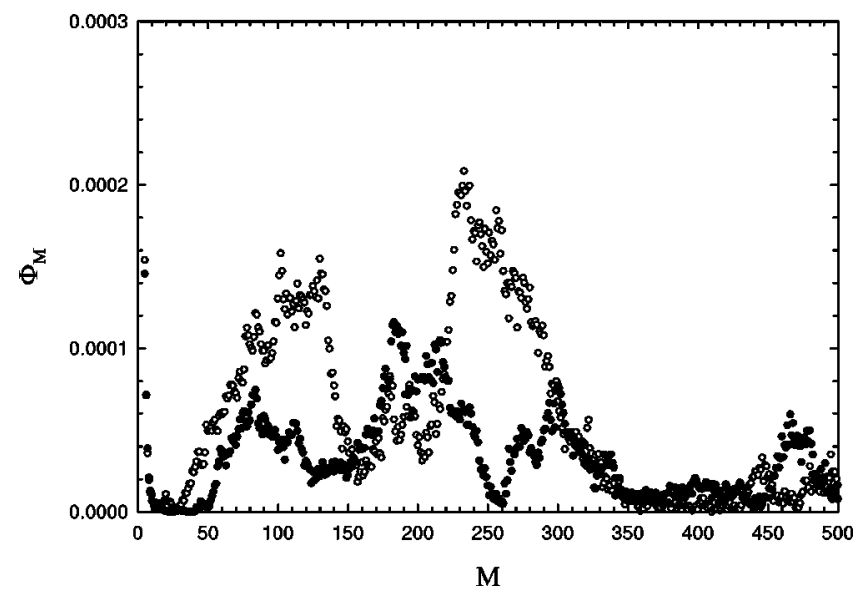

FIG. 6. Aggregate size distributions plotted as volume fraction $\Phi_{M}$ of micelles with the aggregation number $M$ vs $M$ in the unstable region for 10 vol \% (O) and $15 \mathrm{vol} \%(\bullet)$ of $H_{4} T_{7}$ in a supercritical solvent at a reduced temperature of 1.15 and a reduced solvent density of 1.000 .
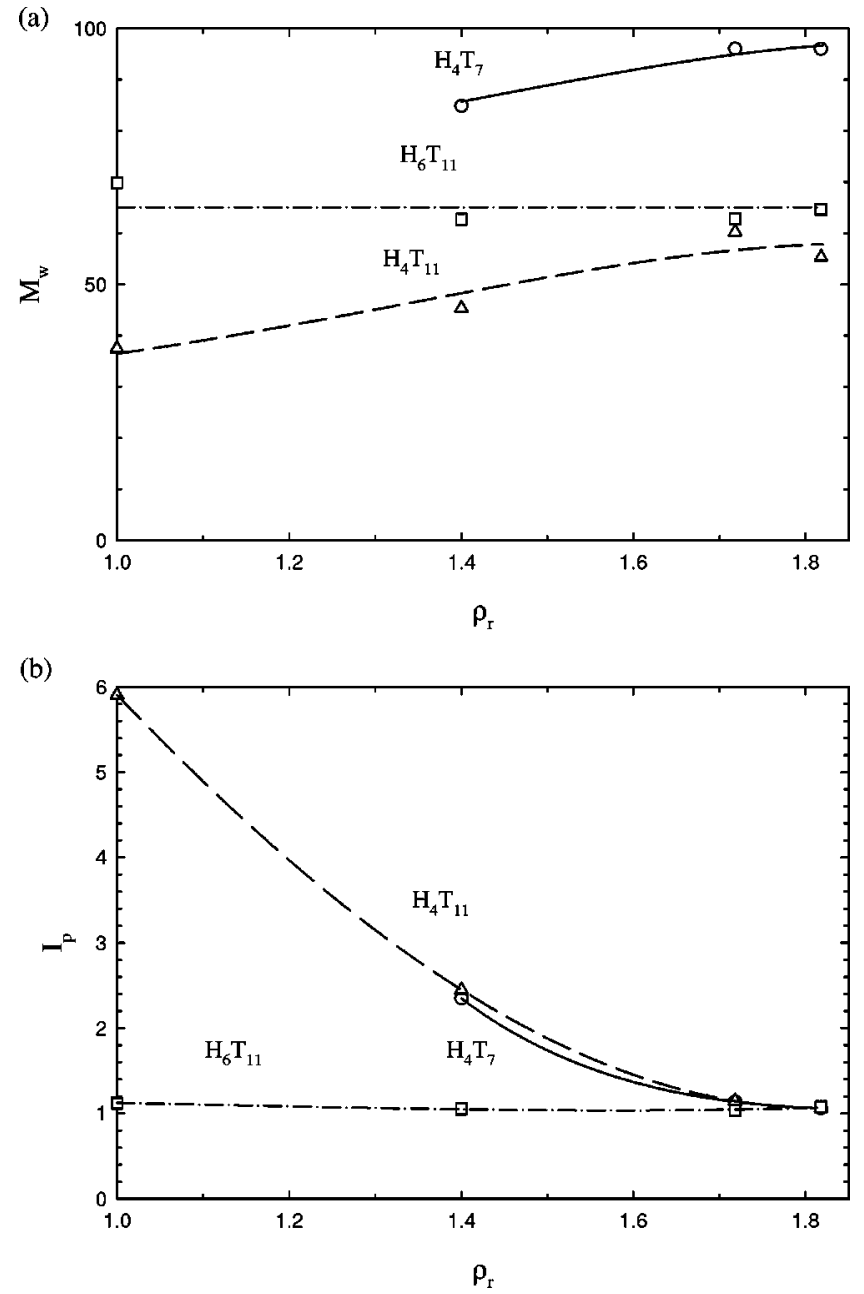

FIG. 7. (a) The weight-average aggregation number $M_{w}$ vs reduced solvent density $\rho_{r}$ and (b) the polydispersity index $I_{p}$ vs $\rho_{r}$ for $3.5 \mathrm{vol} \%$ of $H_{4} T_{7}$ $(\bigcirc,-), 5 \mathrm{vol} \%$ of $H_{4} T_{11}(\triangle,---)$ and $2.5 \mathrm{vol} \%$ of $H_{6} T_{11}(\square,-\cdot-)$ in a supercritical solvent at a reduced temperature of 1.15 .

surfactant with more $H$ segments, $H_{6} T_{11}$. This may explain both the decrease in $M_{w}$ and increase in $I_{p}$ with decreasing $\rho_{r}$ for $H_{4} T_{7}$ and $H_{4} T_{11}$ in contrast to $H_{6} T_{11}$. We also see that at high $\rho_{r}$ the number of surfactant $H$ and $T$ segments has no significant influence on the width of the aggregate size distributions since $I_{p}$ is close to unity for the three surfactants studied.

\section{Critical micelle concentration}

To determine the values of the $\mathrm{cmc}$ at various solvent densities, we plot the mole fraction of free unimers $x_{1}$ vs surfactant mole fraction $x_{A}$ as shown in Fig. 8. Below the cmc where there are no micelles and nearly all surfactants are in the form of free unimers, $x_{1}$ is equal to $x_{A}$ and the simulation points therefore lie on the curve $x_{1}=x_{A}$. Above the cmc, there is a region where $x_{1}$ is a linear decreasing function of $x_{A}$. The linear decrease in $x_{1}$ with increasing $x_{A}$ has already been observed in previous simulations on Larson-type models. ${ }^{29,34,42-44}$ However, we also observe that with the exception of the simulations at $\rho_{r}=1.000$ there is a certain $x_{A}$ above which the slope decreases even more steeply and becomes nonlinear. 

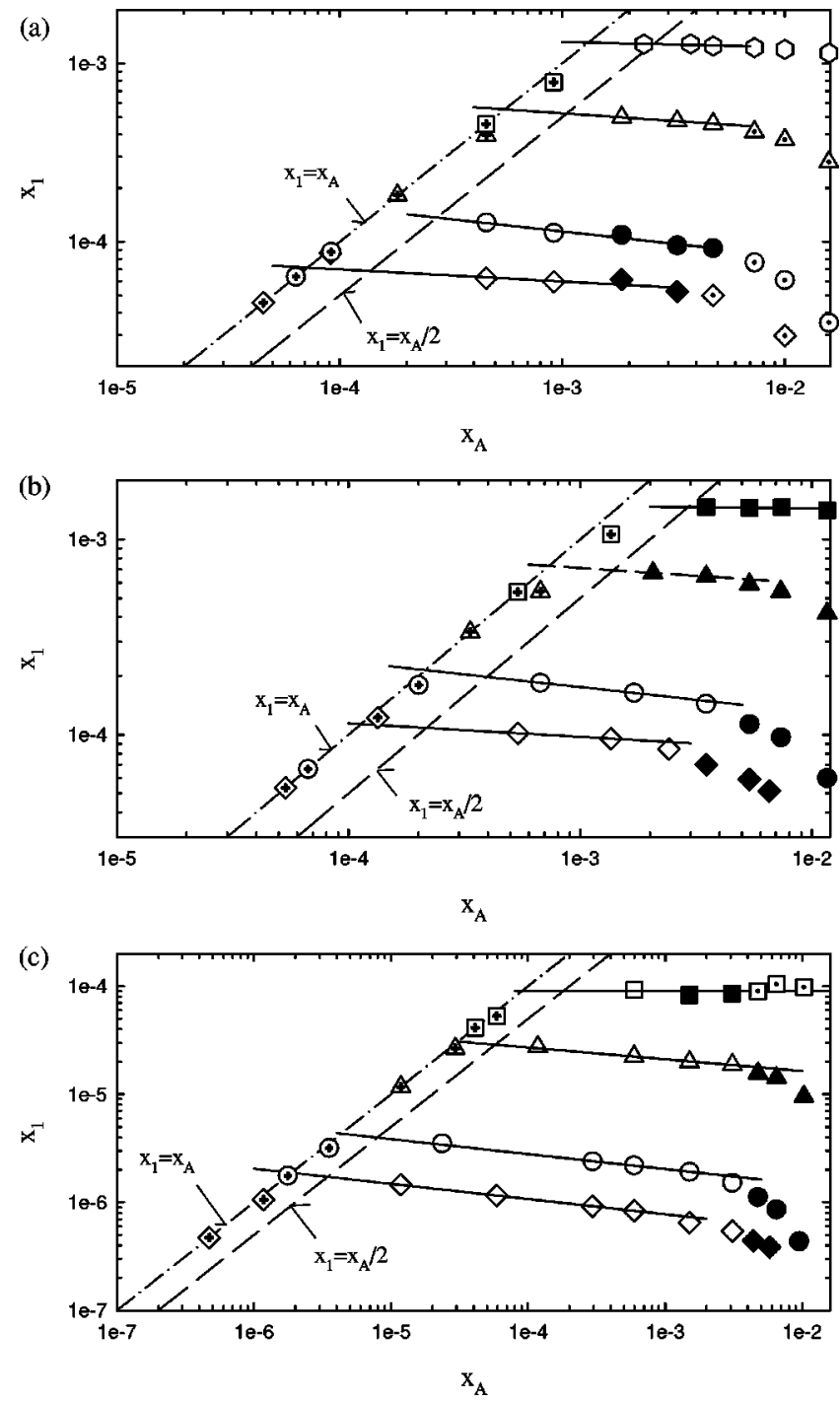

FIG. 8. Mole fraction of free unimers $x_{1}$ vs surfactant mole fraction $x_{A}$ for (a) $H_{4} T_{7}$, (b) $H_{4} T_{11}$, and (c) $H_{6} T_{11}$ in a supercritical solvent at a reduced temperature of 1.15 and reduced solvent densities $\rho_{r}=1.000$ (boxes), 1.400 (triangles), 1.718 (circles), and 1.818 (diamonds). Marks with a cross denote simulation points below the critical micelle concentration $(\mathrm{cmc})$. Open marks represent simulation points above the $\mathrm{cmc}$ where the systems contain spherical micelles. Filled marks correspond to simulation points above the $\mathrm{cmc}$ where the systems contain spherical micelles and growth of the micelles at higher aggregation numbers. Marks with a dot represent simulation points above the $\mathrm{cmc}$ where the systems contain both spherical and elongated micelles. In the case of $H_{4} T_{7}$ at $\rho_{r}=1.000$, hexagons denote the simulation points that fall within the unstable region. The dashed line corresponds to $x_{1}=x_{A} / 2$, the dashed-dotted line represents $x_{1}=x_{A}$, and solid lines are fits of the linear portion of simulation results.

Therefore in Fig. 8, we distinguish simulation state points above the cmc where we observe the spherical micelles (open marks), spherical micelles and growth of the micelles at higher $M$ (filled marks), and both spherical and elongated micelles (marks with a dot) to find if there is a relation between these regions and the decrease in $x_{1}$. In the case of $H_{4} T_{7}$ [Fig. 8(a)], the state points showing spherical micelles and the state points showing spherical micelles and growth of the micelles at higher $M$ fall within the linear portion of the graph. The steeper and nonlinear decrease in $x_{1}$ corresponds to the state points with both spherical and

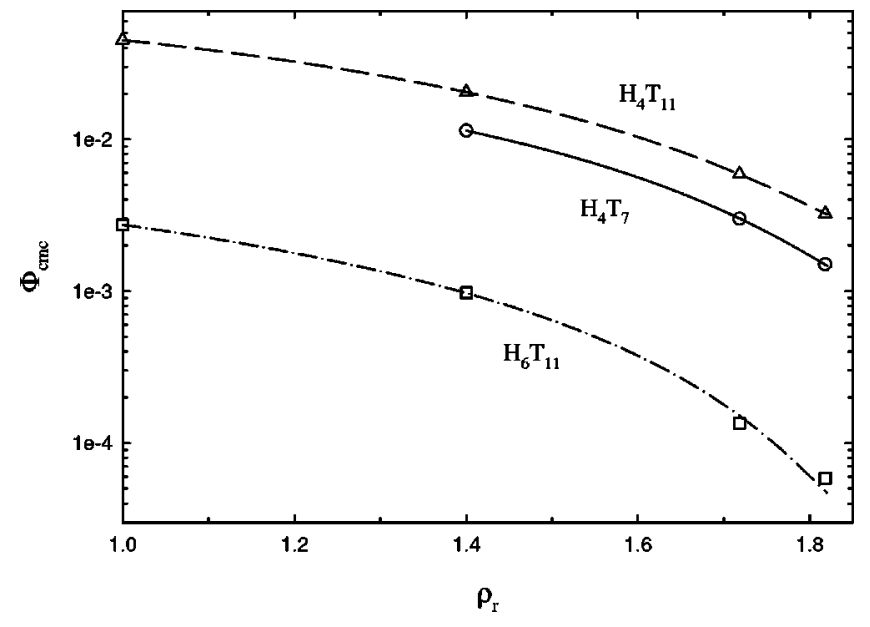

FIG. 9. Critical micelle concentration expressed in volume fraction $\Phi_{\text {cmc }}$ vs reduced solvent density $\rho_{r}$ for $H_{4} T_{7}(\bigcirc,-), H_{4} T_{11}(\triangle,---)$, and $H_{6} T_{11}$ $(\square,-\cdot-)$ in a supercritical solvent at a reduced temperature of 1.15 .

elongated micelles. Note that in the case of $H_{4} T_{7}$ at a low solvent density $\rho_{r}=1.000$, hexagons represent simulation points from an unstable region. In this unstable region, $x_{1}$ also linearly decreases with increasing $x_{A}$ as has already been observed by Girardi and Figueiredo. ${ }^{44}$ In the cases of $H_{4} T_{11}$ [Fig. 8(b)] and $H_{6} T_{11}$ [Fig. 8(c)], especially at higher solvent densities, the state points that are spherical micelles lie on the linear portion of the graph and the steeper and nonlinear decrease in $x_{1}$ is associated with spherical micelles and growth of the micelles at higher $M$. In both cases at a low solvent density $\rho_{r}=1.000$, we see that $x_{1}$ decreases only slightly with increasing $x_{A}$.

We define the $\mathrm{cmc}$ as the surfactant mole fraction at which the number of surfactants aggregated in micelles is equal to the number of free unimers. ${ }^{32}$ The value of the $\mathrm{cmc}$ at various $\rho_{r}$ is determined as the point of intersection of the linear portion of the curve $x_{1}$ vs $x_{A}$ with the curve $x_{1}$ $=x_{A} / 2$. It should be pointed out that in the case of $H_{4} T_{7}$ at $\rho_{r}=1.000$, the intersection coincides roughly with the boundary between the region with free unimers and the unstable region. Figure 9 shows values of the $\mathrm{cmc}$ expressed in volume fraction $\Phi_{\text {cmc }}$ vs $\rho_{r}$. We see from Fig. 9 that the values of the cmc decrease with increasing $\rho_{r}$ for the three surfactants studied. The $\mathrm{cmc}$ for the surfactant with more $H$ segments, $H_{6} T_{11}$, is substantially lower than the cmc's for the surfactants with less $H$ segments, $H_{4} T_{7}$ and $H_{4} T_{11}$. The cmc's for the surfactants with a fixed number of $H$ segments but with a different number of $T$ segments, $H_{4} T_{7}$ and $H_{4} T_{11}$, do not differ significantly from each other-the cmc for $H_{4} T_{11}$ is only slightly higher than that for $H_{4} T_{7}$. The observed dependence of the cmc's on the number of $H$ and $T$ segments agrees with experimental observations for nonionic surfactants alkylpoly(ethylene oxide) in water where the cmc's increase with increasing the poly(ethylene oxide) chain length and decrease with increasing the alkyl chain length (see Figs. 15.15 and 15.16 in Ref. 45).

\section{Pseudophase diagrams}

Based on the analysis of the aggregate size distributions, principal radii of gyration, asphericity parameters and on vi- 

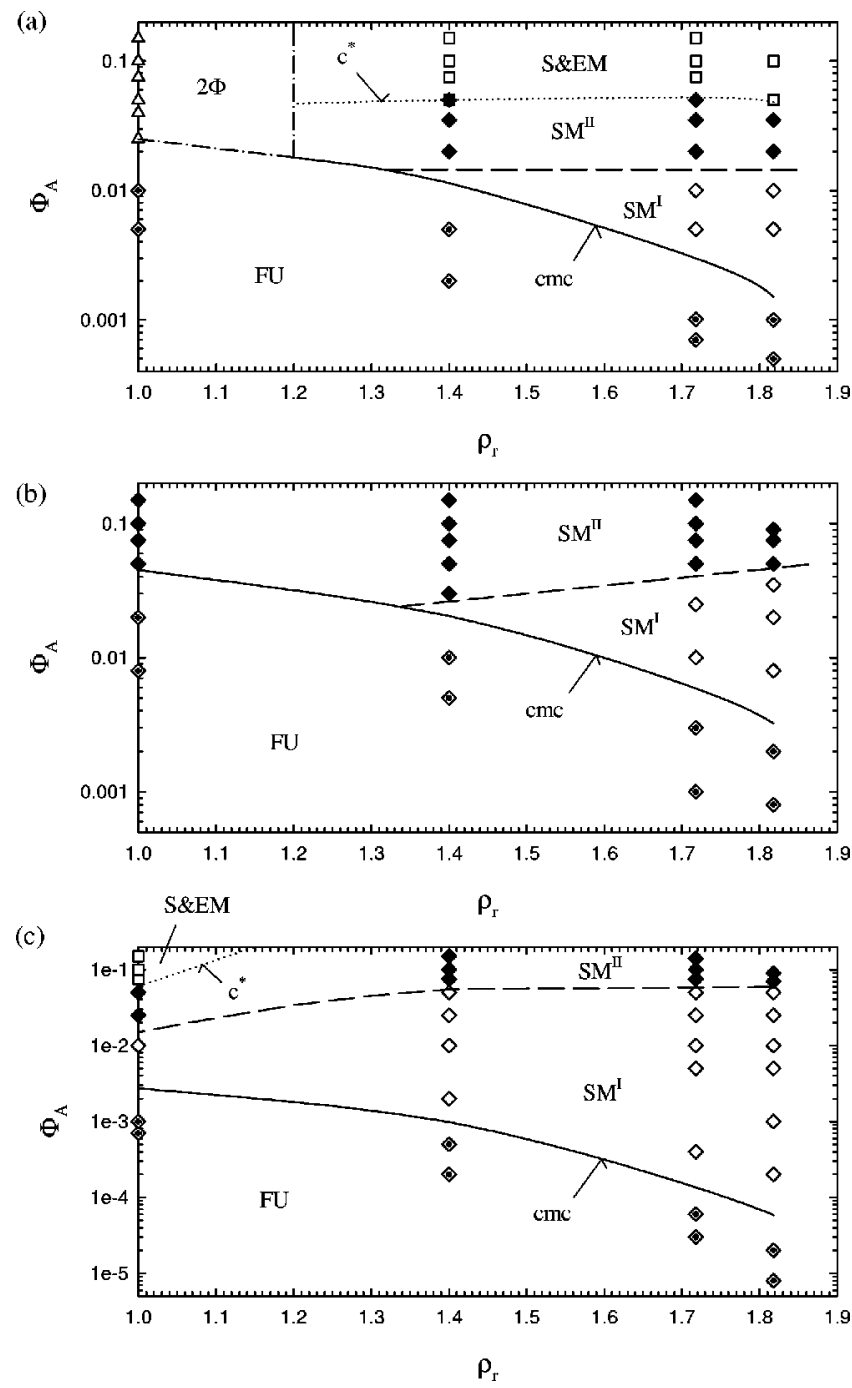

FIG. 10. Pseudophase diagram plotted as surfactant volume fraction $\Phi_{A}$ vs reduced solvent density $\rho_{r}$ at a reduced temperature of 1.15 for (a) $H_{4} T_{7}$, (b) $H_{4} T_{11}$, and (c) $\mathrm{H}_{6} \mathrm{~T}_{11}$ in a supercritical solvent. Marks correspond to simulation state points. Acronyms denote $\mathrm{FU}$, a region containing free unimers; $\mathrm{SM}^{\mathrm{I}}$, a region containing spherical micelles; $\mathrm{SM}^{\mathrm{II}}$, a region containing spherical micelles and growth of the micelles at higher aggregation numbers; S\&EM, a region containing both spherical and elongated micelles; $2 \Phi$, an unstable region; cmc, critical micelle concentration; and $c^{*}$, overlap concentration.

sual inspection of simulation snapshots, we construct pseudophase diagrams for the three model systems studied at $T_{r}=1.15$. These are plotted in $\Phi_{A}-\rho_{r}$ space in Fig. 10.

In the pseudophase diagrams, we identify various regions according to the number of peaks in the aggregate size distributions. In the case of the $H_{4} T_{7}$ system [Fig. 10(a)], the pseudophase diagram exhibits four regions: (i) a region containing free unimers (FU), (ii) a region containing spherical micelles (SM) (the cmc is the boundary between the FU and SM regions), (iii) a region containing both spherical and elongated micelles (S\&EM) [the overlap concentration $c^{*}$ (Refs. 46 and 47) is the boundary between the SM and S\&EM regions], and (iv) an unstable region $(2 \Phi)$ at high $\Phi_{A}$ and low $\rho_{r}$. Aggregate size distributions for state points from the FU region have one (unimeric) peak and aggregate size distributions for state points from the SM region have two (unimeric and micellar) peaks. Aggregate size distributions for state points from the S\&EM region have multiple (unimeric and several micellar) peaks while aggregate size distributions for state points from the $2 \Phi$ region have multiple size-dependent and irregularly distributed peaks. The SM region is further divided into two subregions denoted by $\mathrm{SM}^{\mathrm{I}}$ and $\mathrm{SM}^{\mathrm{II}}$. Systems from the $\mathrm{SM}^{\mathrm{I}}$ subregion contains only spherical micelles; the aggregate size distributions are symmetrical around the micellar peak and $R_{1} \approx R_{2} \approx R_{3}$. Systems from the $\mathrm{SM}^{\mathrm{II}}$ subregion contains spherical micelles at lower $M$ and growth of the micelles at higher $M$; the aggregate size distributions are asymmetrical around the micellar peak, and $R_{1} \approx R_{2} \approx R_{3}$ at lower $M$ and $R_{1}$ increases in contrast to $R_{2}$ and $R_{3}$ with increasing $M$ at higher $M$. It should be pointed out that the overlap concentration and boundaries between the $\mathrm{SM}^{\mathrm{I}}$ and $\mathrm{SM}^{\mathrm{II}}$ subregions, between the SM and $2 \Phi$ regions and between the S\&EM and $2 \Phi$ regions are drawn by hand as a guide for the eye only.

The pseudophase diagram for the $H_{4} T_{11}$ system [Fig. 10(b)] shows, in contrast to the $H_{4} T_{7}$ system, only two regions: (i) a FU region and (ii) a $\mathrm{SM}$ region (with $\mathrm{SM}^{\mathrm{I}}$ and $\mathrm{SM}^{\mathrm{II}}$ subregions). The pseudophase diagram for the $H_{6} T_{11}$ system [Fig. 10(c)] is similar to the pseudophase diagram for the $H_{4} T_{11}$ system with an additional S\&EM region at high $\Phi_{A}$ and low $\rho_{r}$.

It is interesting to compare the model pseudophase diagrams with those obtained from experiment. Figure 11(a) shows the pseudophase diagram for diblock copolymer PVAC-PTAN in $\mathrm{scCO}_{2}$ at $T_{r}=1.03$ measured using static and dynamic light scattering by Buhler et al. ${ }^{46}$ The experimental pseudophase diagram has some qualitative features in common with the model pseudophase diagram for the $H_{4} T_{7}$ system [Fig. 10(a)]. In both the experimental and model pseudophase diagrams, we see the FU, SM, S\&EM, and $2 \Phi$ regions. However, the pseudophase diagrams differ in the density dependence of the cmc. The experimental pseudophase diagram shows an increase in the $\mathrm{cmc}$ with increasing $\rho_{r}$ while the model pseudophase diagrams shows a decrease in the cmc with increasing $\rho_{r}$. Experimental investigation of the effect of increasing pressure $P$ on the cmc (Ref. 48) of nonionic surfactant Triton-X-100 and ionic surfactants such as cationic sodium dodecylsulfate and anionic decyltrimethylammonium bromide in aqueous solutions shows both trends, an increase in the cmc with increasing $P$ at lower $P$ and a decrease in the cmc with increasing $P$ at higher $P$ as shown in Fig. 11(b) for the Triton-X-100 in water at $295 \mathrm{~K}^{48}$

Buhler et al. ${ }^{46}$ explain the increase in the cmc with increasing $\rho_{r}$ for the PVAC-PTAN system as follows. In the PVAC-PTAN diblock copolymer, PVAC is the $\mathrm{CO}_{2}$-phobic block while PTAN is the $\mathrm{CO}_{2}$-philic block. At low $\rho_{r}$, where $\mathrm{CO}_{2}$ is a bad solvent for the PVAC block but a good solvent for the PTAN block, the spherical micelles form at a certain copolymer concentration due to different solvating ability of $\mathrm{scCO}_{2}$ for the PVAC and PTAN blocks. As $\rho_{r}$ increases, the solvent quality for both blocks become better, increasing the solubility of the PVAC and PTAN blocks in $\mathrm{scCO}_{2}$ as confirmed by cloud-curve measurements. ${ }^{46}$ As a result, more copolymers need to be added to the solution to 
(a)

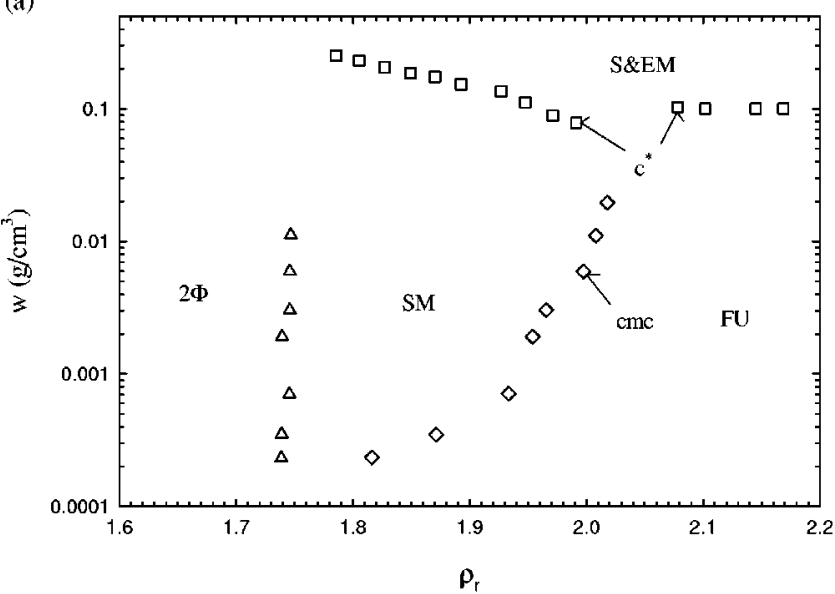

(b)

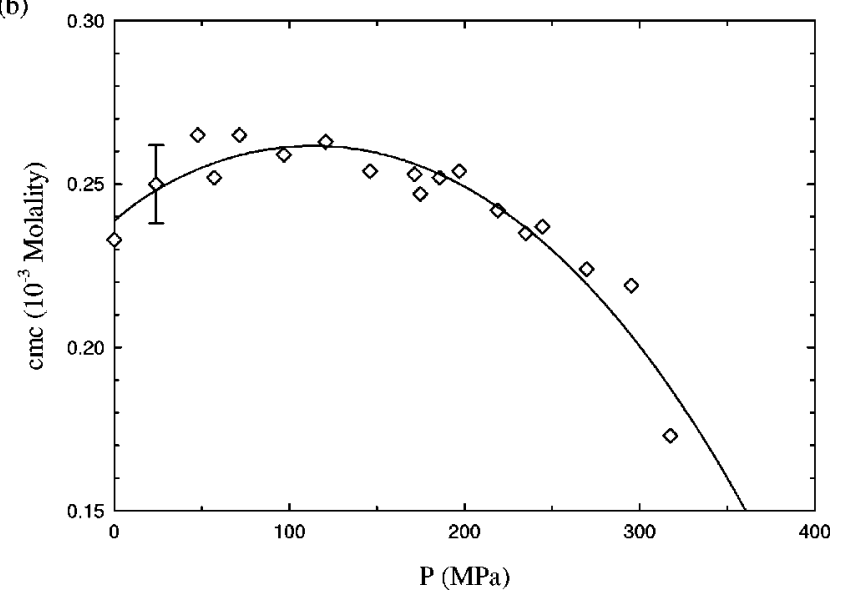

FIG. 11. (a) Pseudophase diagram plotted as surfactant mass concentration $w$ vs reduced solvent density $\rho_{r}$ at a reduced temperature of 1.03 for the diblock copolymer PVAC-PTAN in supercritical carbon dioxide. Points correspond to experimental results (Ref. 46). Acronyms denote FU, a region containing free unimers; SM, a region containing spherical micelles; S\&EM, a region containing both spherical and elongated micelles; $2 \Phi$, an unstable region; cmc, critical micelle concentration; and $c^{*}$, overlap concentration. (b) The cmc vs pressure $P$ for nonionic surfactant Triton-X-100 in water at 295 K. Diamonds correspond to experimental results (Ref. 48) and the solid line represents a fit of the experimental results.

form micelles and the cmc increases with increasing $\rho_{r}$. Using similar arguments, we may justify the decrease in the cmc with increasing $\rho_{r}$ observed in the model supercritical solvent $-H_{m} T_{n}$ systems. In the case of surfactant $H_{m} T_{n}, H_{m}$ is the solvent-phobic part of the surfactant and $T_{n}$ is the solvent-philic part of the surfactant. At low $\rho_{r}$, the solvent is a bad solvent for the $H_{m}$ chain and a good solvent for the $T_{n}$ chain, and the spherical micelles form at a certain surfactant concentration. Our calculations based on the lattice fluid theory ${ }^{17}$ predict (data not shown) that at $T_{r}=1.15$, the solubility of the homopolymer chain $H_{m}$ in the solvent decreases with increasing $\rho_{r}$ while the homopolymer chain $T_{n}$ is completely soluble in the solvent within the range of $\rho_{r}$ studied. As a result, less surfactant needs to be added to the solution to form micelles as $\rho_{r}$ increases and hence, the cmc decreases with increasing $\rho_{r}$.

\section{B. Micellar solubilization}

We measure the ability of the surfactant solutions to dissolve more solutes than the solutions without surfactants by calculating the partition coefficient $K$ using the two-box simulation method (Sec. IV B 3). The simulations are carried out at $T_{r}=1.15$ and $\rho_{r}=1.718$; such a density is typically used in real $\mathrm{scCO}_{2}$-surfactant systems. ${ }^{46}$ We keep the overall surfactant concentration equal to $2.5 \mathrm{vol} \%$ and vary the overall solute concentration $z_{l}$ from 1 vol $\%$ to 16 vol $\%$. The number of particle transfers used is typically from 1000 to 2000 per MC cycle. At each simulated state point, we measure the concentration of the species in both boxes, the aggregate size distribution and the three principal radii of gyration $\left\{R_{1}, R_{2}, R_{3}\right\}$. The partition coefficient is subsequently evaluated from Eq. (4.8). To measure the aggregate size distributions and principal radii of gyration, we need to modify the computational definition of an aggregate previously introduced for solvent-surfactant systems to account for the presence of solute molecules. An aggregate is defined as a collection of surfactant head segments and solute molecules that are nearest or diagonal nearest neighbors each other. It is no longer necessary for all surfactant head segments to touch each other directly. The aggregation number is still defined as the number of surfactant chains in the aggregate. Further, any solute that either has at least one nearest or diagonal nearest neighbor contact with a head segment in a micelle or has a neighbor solute with such a contact is considered to be a solubilized solute. All other solutes are considered to be unsolubilized solutes. ${ }^{34}$

In Fig. 12, we present $K$ vs volume fraction of solute in the solvent box $\Phi_{l}^{s}$ [Fig. 12(a)] and the solute/surfactant ratio $\Phi_{l}^{A} / \Phi_{A}^{A}$ vs $\Phi_{l}^{s}$ [Fig. 12(b)], where $\Phi_{l}^{A}$ and $\Phi_{A}^{A}$ are, respectively, the solute and surfactant volume fractions in the micelle box. Our calculation based on the lattice fluid theory ${ }^{17}$ gives a value of $2.3 \%$ for the solubility limit of the solute in the solvent box. From Fig. 12, we see that for all simulation points, $\Phi_{l}^{s}$ is below this limit and hence, the micelle box contains a stable phase. Furthermore, we see that for the three systems studied $K$ and $\Phi_{l}^{A} / \Phi_{A}^{A}$ increase only slightly with increasing $\Phi_{l}^{s}$ up to about $1.4 \%$. This is followed by a sharp increase in $K$ and $\Phi_{l}^{A} / \Phi_{A}^{A}$ at the larger $\Phi_{l}^{s}$. Based on visual inspection of the simulation snapshots (not shown) we have found that in the region with the slight increase of $K$ and $\Phi_{l}^{A} / \Phi_{A}^{A}$ as a function of $\Phi_{l}^{s}$, solubilized solute molecules are present entirely within the region of the surfactant head blocks (called in Ref. 45 Type I solubilization). In the region with the sharp increase of $K$ and $\Phi_{l}^{A} / \Phi_{A}^{A}$ as a function of $\Phi_{l}^{s}$, solubilized solute molecules are present in a domain containing a pure solute phase (droplet) formed inside the micellar core in addition to being present in the region of the surfactant head blocks (called in Ref. 45 Type II solubilization).

Figure 13 shows the aggregate size distributions and three principal radii of gyration $\left\{R_{1}, R_{2}, R_{3}\right\}$ vs aggregation number $M$ for the solute containing $H_{4} T_{11}$ system at various overall solute concentrations $z_{l}$. We see that aggregate size distributions [Fig. 13(a)] and $\left\{R_{1}, R_{2}, R_{3}\right\}$ vs $M$ [Fig. 13(b)] for the system without solute $\left(z_{l}=0 \mathrm{vol} \%\right)$ and for the system with the type I solubilization $\left(z_{l}=4 \mathrm{vol} \%\right)$ do not differ significantly. In contrast, the systems with the type II solubilization exhibit dramatic growth of the size of micelles due to the formation of a droplet in the micellar core, as con- 

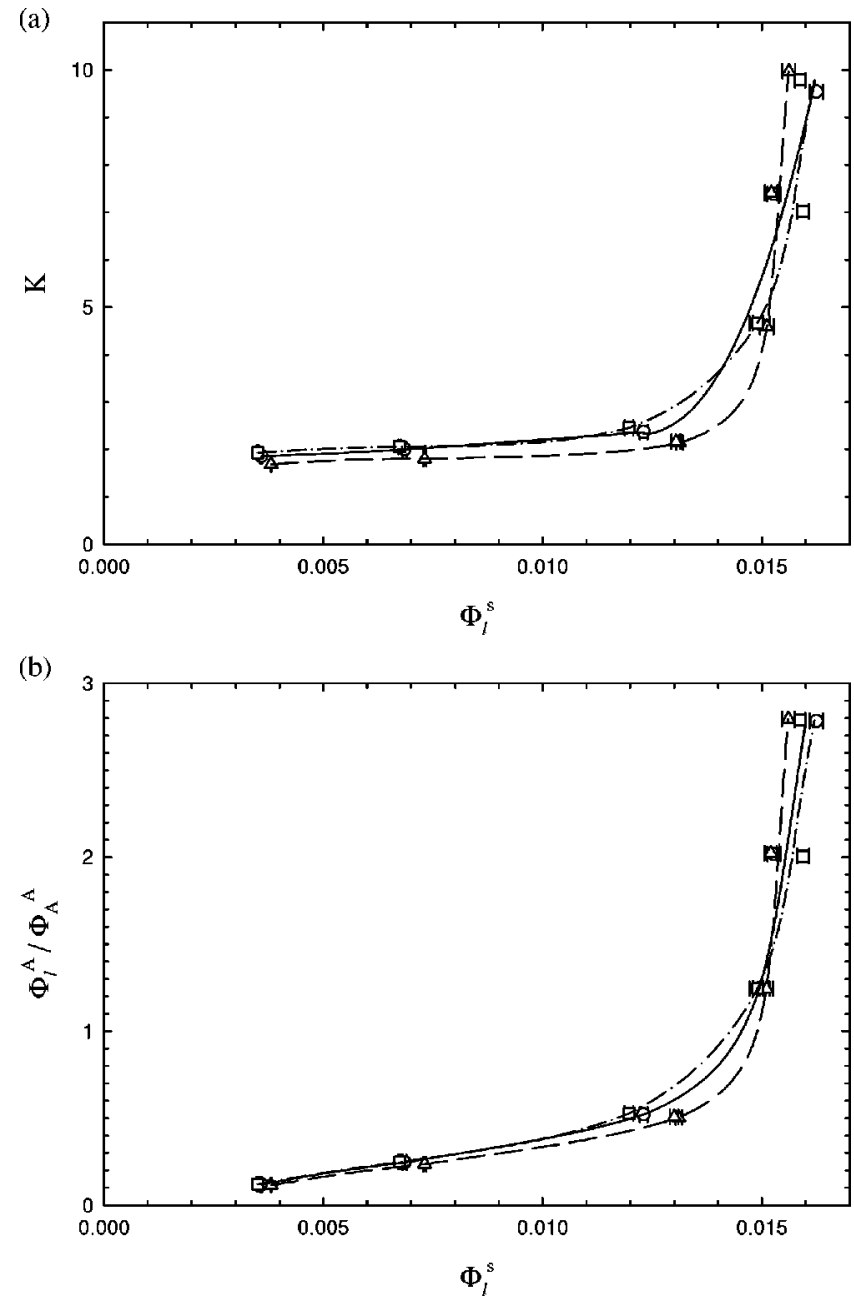

FIG. 12. (a) The partition coefficient $K$ vs solute volume fraction in the solvent box $\Phi_{l}^{s}$ and (b) the solute/surfactant ratio $\Phi_{l}^{A} / \Phi_{A}^{A}$ vs $\Phi_{l}^{s}\left(\Phi_{l}^{A}\right.$ and $\Phi_{A}^{A}$ are, respectively, solute and surfactant volume fractions in the micelle box) for $H_{4} T_{7}(\bigcirc,-), H_{4} T_{11}(\triangle,---)$, and $H_{6} T_{11}(\square,-\cdot-)$ systems at a reduced temperature of 1.15 and a reduced solvent density of 1.718 .

firmed by a shift of the micellar peak to higher $M$ [Fig. 13(a), $z_{l}=8 \mathrm{vol} \%$ and $\left.16 \mathrm{vol} \%\right]$ and by an increase of values for $R_{1}, R_{2}$, and $R_{3}$ [Fig. 13(b), $z_{l}=8$ vol $\%$ and 16 vol \%]. The solute containing $H_{4} T_{7}$ and $H_{6} T_{11}$ systems show similar behavior (data not shown).

The behavior described in Figs. 12 and 13 can be understood as follow. The head-head segment and solute-solute contact energies are $\varepsilon_{H H} / k_{B}=-71 \mathrm{~K}$ and $\varepsilon_{l l} / k_{B}$ $=-111 \mathrm{~K}$, respectively. At a lower solute concentration, solubilized solute molecules are distributed within the region of the surfactant head blocks since there are many more contacts between head-head segments than between solutesolute molecules. As solute concentration increases, the number of solute-solute contacts also increases and the region of the surfactant head blocks becomes saturated by the solute molecules. Since $\left|\varepsilon_{l l}\right|>\left|\varepsilon_{H H}\right|$, the solute molecules start to self-organize inside the micellar core and form a droplet. Further, due to $\left|\varepsilon_{l l}\right|>\left|\varepsilon_{H H}\right|$ the solute molecules accommodate much more easily into the droplet than into the region of the surfactant head blocks. Hence, the systems with the type II solubilization exhibit much greater increase in the partition

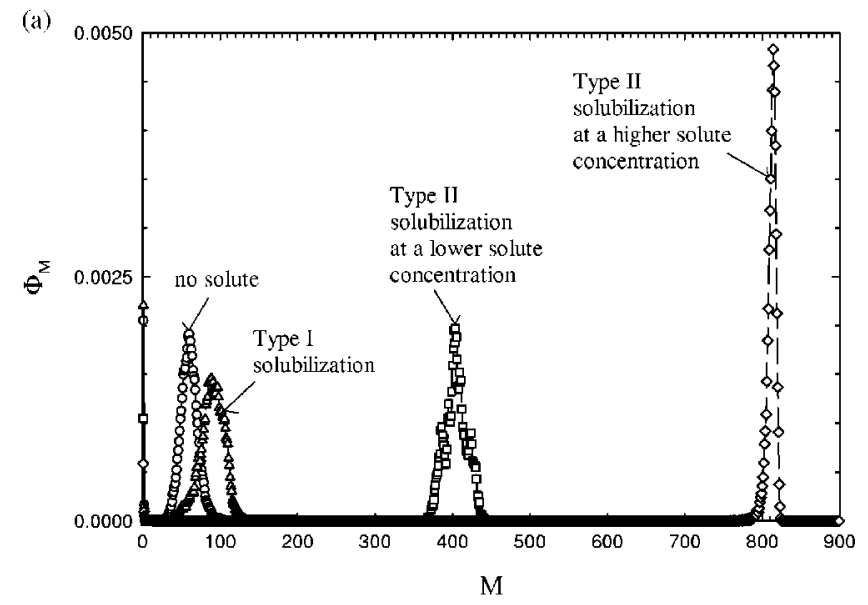

(b)

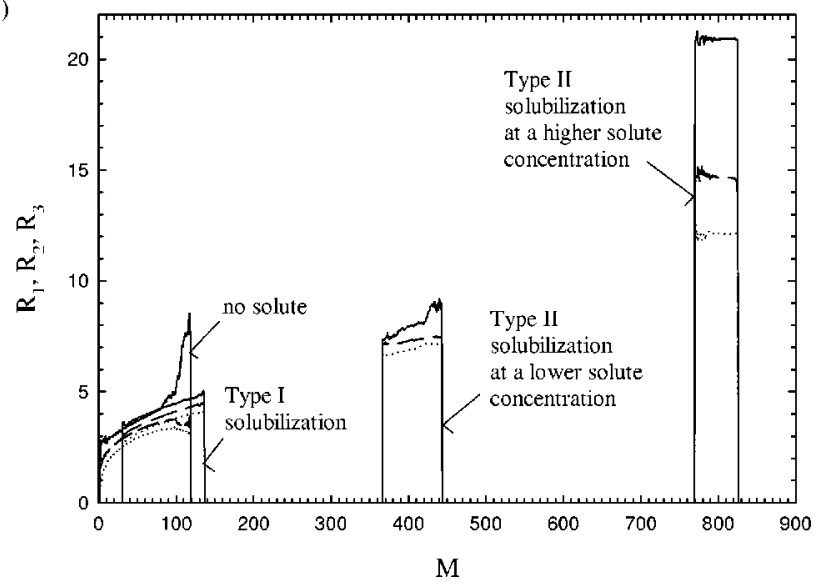

FIG. 13. (a) Aggregate size distributions vs aggregation number $M$ at various overall solute concentrations $z_{l}: z_{l}=0$ vol $\%(\bigcirc), z_{l}=4$ vol \% ( $\left.\triangle\right)$, $z_{l}=8 \mathrm{vol} \%(\square), z_{l}=16 \mathrm{vol} \%(\diamond)$ and $(\mathrm{b})$ three principal radii of gyration $R_{1}(-), R_{2}(---), R_{3}(\cdots)$ vs $M$ corresponding to these values of $z_{l}$ for $H_{4} T_{11}$ system at a reduced temperature of 1.15 and a reduced solvent density of 1.718 .

coefficient, the solute/surfactant ratio and the size of micelles with increasing solute concentration than the systems with the type I solubilization.

\section{CONCLUSIONS}

We studied self-assembly of a nonionic surfactant perfluoroalkylpoly(ethylene oxide): $\mathrm{F}\left(\mathrm{CF}_{2}\right)_{n}\left(\mathrm{CH}_{2} \mathrm{CH}_{2} \mathrm{O}\right)_{m} \mathrm{H}(m$ $=4-9, n=7-11 ; m<n)$ in supercritical carbon dioxide $\left(\mathrm{scCO}_{2}\right)$ using a modified version of Larson's lattice model, focusing on $H_{4} T_{7}, H_{4} T_{11}$, and $H_{6} T_{11}$ to observe the effect of tail- and head-length variations. We performed canonical Monte Carlo simulations at the reduced temperature $T_{r}$ of 1.15 , varying the reduced solvent densities $\rho_{r}$ from 1 to 1.82 and the surfactant volume fractions $\Phi_{A}$ from $0.1 \mathrm{vol} \%$ to 15 vol \%. At each simulation state point, we calculated the aggregate size distribution, the three principal radii of gyration and the asphericity parameter. The critical micelle concentration $(\mathrm{cmc})$ was determined as the concentration at which the number of surfactants aggregated in micelles is equal to the number of free unimers. ${ }^{32}$ We found that the $\mathrm{cmc}$ for the surfactant with more $H$ segments, $H_{6} T_{11}$, was substantially lower than the cmc's for the surfactants with less $H$ seg- 
ments, $H_{4} T_{7}$ and $H_{4} T_{11}$. The cmc's for the surfactants with a fixed number of $H$ segments but with a different number of $T$ segments, $H_{4} T_{7}$ and $H_{4} T_{11}$, did not differ significantly from each other. The observed dependence of the cmc's on the number of $H$ and $T$ segments agreed with experimental observations for nonionic surfactants alkylpoly(ethylene oxide) in water. Further, we observed that the cmc's for the three systems studied decreased with increasing the solvent density. In contrast, experimental investigation of the PVAC-PTAN- $\mathrm{scCO}_{2}$ system ${ }^{46}$ showed an increase in the $\mathrm{cmc}$ with increasing solvent density. We explained different density dependence of the $\mathrm{cmc}$ found in the model systems and the PVAC-PTAN- $\mathrm{scCO}_{2}$ system by different solubility behavior of surfactant head blocks in $\mathrm{scCO}_{2}$ when changing the solvent density.

From simulation data, we constructed pseudophase diagrams for the $H_{4} T_{7}, H_{4} T_{11}$, and $H_{6} T_{11}$ systems. The pseudophase diagram for $H_{4} T_{7}$ system exhibits four regions: (i) a region containing free unimers (FU), (ii) a region containing spherical micelles (SM), (iii) a region containing both spherical and elongated micelles (S\&EM), and (iv) an unstable region $(2 \Phi)$ at higher surfactant concentration and low solvent density. The pseudophase diagram for $H_{4} T_{11}$ system showed, in contrast to the $H_{4} T_{7}$ system, only two regions: (i) a FU region and (ii) a $\mathrm{SM}$ region. The pseudophase diagram for the $H_{6} T_{11}$ system was similar to the pseudophase diagram for the $H_{4} T_{11}$ system with an additional S\&EM region at higher surfactant concentration and low solvent density. The pseudophase diagram for the $H_{4} T_{7}$ system had some qualitative features in common with the experimental pseudophase diagram for the PVAC-PTAN- $\mathrm{scCO}_{2}$ system. ${ }^{46}$ In both the model and experimental pseudophase diagrams, we saw the FU, SM, S\&EM, and $2 \Phi$ regions.

We measured the ability of the surfactant solutions to dissolve more solutes than solutions without surfactants by calculating the partition coefficient using the two-box simulation method, which is a computational analog of a real osmosis experiment. Water served as a prototype of the solute. We found that at lower solute concentration, the solubilized solute molecules were present entirely within the region of the surfactant head blocks, and the partition coefficient and solute/surfactant ratio increased only slightly with increasing the solute concentration. At larger solute concentration, the solubilized solute molecules were present as a droplet inside the micellar core in addition to being present in the region of the surfactant head blocks, and the partition coefficient and solute/surfactant ratio increased dramatically with increasing the solute concentration.

\section{ACKNOWLEDGMENTS}

The material is based upon work supported by the STC Program of the National Science Foundation under Agreement No. CHE-9876674 and by the Grant Agency of the Czech Republic (Grant No. 203/00/0600). M.L. thanks Flor R. Siperstein for fruitful discussions and many valuable comments.
${ }^{1}$ M. McHugh and V. Krukonis, Supercritical Fluid Extraction (Butterworths, Boston, 1986).

${ }^{2}$ J. M. DeSimone, Z. Guan, and C. S. Elsbernd, Science 257, 945 (1992).

${ }^{3}$ http://www.nsfstc.unc.edu

${ }^{4}$ G. G. Yee, J. L. Fulton, and R. D. Smith, J. Phys. Chem. 96, 6172 (1992).

${ }^{5}$ A. Iezzi, P. Bendale, R. M. Enick, M. Turberg, and J. Brady, Fluid Phase Equilibria 52, 307 (1989).

${ }^{6}$ S. Salaniwal, S. T. Cui, P. T. Cummings, and H. D. Cochran, Langmuir 15, 5188 (1999).

${ }^{7}$ S. Salaniwal, S. T. Cui, H. D. Cochran, and P. T. Cummings, Ind. Eng. Chem. Res. 39, 4543 (2000).

${ }^{8}$ S. Salaniwal, S. T. Cui, H. D. Cochran, and P. T. Cummings, Langmuir 17, 1773 (2001); 17, 1784 (2001).

${ }^{9}$ C. Baysal, B. Erman, and B. Chu, J. Chem. Phys. 114, 5444 (2001).

${ }^{10}$ G. Luna-Bárcenas, S. Mawson, S. Takishima, J. M. DeSimone, I. C. Sanchez, and K. P. Johnston, Fluid Phase Equilibria 146, 325 (1998).

${ }^{11}$ W. G. Chapman, K. E. Gubbins, G. Jackson, and M. Radosz, Ind. Eng. Chem. Res. 29, 1709 (1990).

${ }^{12}$ C. M. Colina, C. K. Hall, and K. E. Gubbins, Fluid Phase Equilibria (submitted).

${ }^{13}$ J. C. Shelley and M. Y. Shelley, Curr. Opin. Colloid Interface Sci. 5, 101 (2000).

${ }^{14}$ R. G. Larson, L. E. Scriven, and H. T. Davis, J. Chem. Phys. 83, 2411 (1985).

${ }^{15}$ R. G. Larson, J. Phys. II 6, 1441 (1996).

${ }^{16}$ J. L. Fulton, D. M. Pfund, J. B. McClain, T. J. Romack, E. E. Maury, J. R. Combes, E. T. Samulski, J. M. DeSimone, and M. Capel, Langmuir 11, 4241 (1995).

${ }^{17}$ I. A. Sanchez and C. G. Panayiotou, in Models for Thermodynamic and Phase Equilibria Calculations, edited by S. I. Sandler (Marcel Deker, New York, 1994).

${ }^{18} \mathrm{H}$. Tompa, Polymer Mixtures (Butterworths, London, 1956).

${ }^{19}$ P. J. Flory, Principles of Polymer Chemistry (Cornell University Press, New York, 1953).

${ }^{20}$ A. K. Rappé, C. J. Casewit, K. S. Colwell, W. A. Goddard III, and W. M. Skiff, J. Am. Chem. Soc. 114, 10024 (1992).

${ }^{21}$ R. C. Reid, J. M. Prausnitz, and B. E. Poling, The Properties of Gases and Liquids (McGraw-Hill, New York, 1987).

${ }^{22}$ A. D. Mackie, A. Z. Panagiotopoulos, and S. K. Kumar, J. Chem. Phys. 102, 1014 (1995).

${ }^{23}$ M. P. Allen and D. J. Tildesley, Computer Simulation of Liquids (Clarendon, Oxford, 1987).

${ }^{24}$ F. S. Bates and G. H. Fredrickson, Phys. Today 52, 32 (1999)

${ }^{25}$ F. S. Bates, Science 251, 898 (1991).

${ }^{26}$ J. M. Smith, H. C. Van Ness, and M. M. Abbott, Introduction to Chemical Engineering Thermodynamics (McGraw-Hill, New York, 1996).

${ }^{27}$ A. F. M. Barton, CRC Handbook of Solubility Parameters and Other Cohesion Parameters (CRC Press, Boca Raton, 1983).

${ }^{28}$ M. Nieves García-Lisbona, A. Galindo, G. Jackson, and A. N. Burgess, Mol. Phys. 93, 57 (1998).

${ }^{29}$ A. D. Mackie, A. Z. Panagiotopoulos, and I. Szleifer, Langmuir 13, 5022 (1997).

${ }^{30}$ D. Frenkel and B. Smit, Understanding Molecular Simulation: From Algorithms to Applications (Academic, London, 1996).

${ }^{31}$ J. Hoshen and R. Kopelman, Phys. Rev. B 14, 3438 (1976).

${ }^{32}$ J. N. Israelachvili, D. J. Mitchell, and B. W. Ninham, J. Chem. Soc., Faraday Trans. 2 72, 1525 (1976).

${ }^{33}$ M. A. Floriano, E. Caponetti, and A. Z. Panagiotopoulos, Langmuir 15, 3143 (1999)

${ }^{34}$ S. K. Talsania, Y. Wang, R. Rajagopalan, and K. K. Mohanty, J. Colloid Interface Sci. 190, 92 (1997).

${ }^{35}$ S. K. Talsania, L. A. Rodríguez-Guadarrama, K. K. Mohanty, and R. Rajagopalan, Langmuir 14, 2684 (1998).

${ }^{36}$ P. H. Nelson, T. A. Hatton, and G. C. Rutledge, J. Chem. Phys. 110, 9673 (1999).

${ }^{37}$ S. M. Walas, Phase Equilibria in Chemical Engineering (Butterworths, Boston, 1985).

${ }^{38}$ A. Z. Panagiotopoulos, N. Quirke, M. Stapleton, and D. J. Tildesley, Mol. Phys. 63, 527 (1988).

${ }^{39}$ A. Z. Panagiotopoulos, Int. J. Thermophys. 10, 447 (1992).

${ }^{40}$ D. M. Tsangaris and P. D. McMahon, Mol. Simul. 9, 223 (1992). 
${ }^{41}$ M. Mehta and D. A. Kofke, Mol. Phys. 79, 39 (1993).

${ }^{42}$ L. A. Rodríguez-Guadarrama, S. K. Talsania, K. K. Mohanty, and R. Rajagopalan, Langmuir 15, 437 (1999).

${ }^{43}$ J. C. Desplat and C. M. Care, Mol. Phys. 87, 441 (1996).

${ }^{44}$ M. Girardi and W. Figueiredo, J. Chem. Phys. 112, 4833 (2000).

${ }^{45}$ R. Nagarajan and E. Ruckenstein, "Self-Assembled Systems," in Equations of State for Fluids and Fluid Mixtures, edited by J. V. Sengers, R. F.
Kayser, C. J. Peters, and H. J. White, Jr. (Elsevier, Amsterdam, The Netherlands, 2000).

${ }^{46}$ E. Buhler, A. V. Dobrynin, J. M. DeSimone, and M. Rubinstein, Macromolecules 31, 7347 (1998).

${ }^{47}$ A. Triolo, F. Triolo, F. Lo Celso, D. E. Betts, J. B. McClain, J. M. DeSimone, G. D. Wignall, and R. Triolo, Phys. Rev. E 62, 5839 (2000).

${ }^{48}$ K. Hara, H. Kuwabara, O. Kajimoto, and K. Bhattacharyya, J. Photochem. Photobiol., A 124, 159 (1999). 